\title{
HMGB1 links chronic liver injury to progenitor responses and hepatocarcinogenesis
}

\author{
Celine Hernandez, ${ }^{1}$ Peter Huebener, ${ }^{1,2}$ Jean-Philippe Pradere, ${ }^{3}$ Daniel J. Antoine, ${ }^{4}$ Richard A. Friedman, ${ }^{5}$ and Robert F. Schwabe ${ }^{1}$ \\ 'Department of Medicine, Columbia University, New York, New York, USA. ²Department of Medicine, University Medical Center Hamburg-Eppendorf, Hamburg, Germany. Institut National de la Santé et de la \\ Recherche Médicale (INSERM) U1048, Institute of Cardiovascular and Metabolic Disease, Toulouse, France. ${ }^{4}$ MRC Centre for Inflammation Research, University of Edinburgh, United Kingdom. \\ ${ }^{5}$ Biomedical Informatics Shared Resource, Herbert Irving Comprehensive Cancer Center and Department of Biomedical Informatics, Columbia University, New York, New York, USA.
}

\begin{abstract}
Cell death is a key driver of disease progression and carcinogenesis in chronic liver disease (CLD), highlighted by the wellestablished clinical correlation between hepatocellular death and risk for the development of cirrhosis and hepatocellular carcinoma (HCC). Moreover, hepatocellular death is sufficient to trigger fibrosis and HCC in mice. However, the pathways through which cell death drives CLD progression remain elusive. Here, we tested the hypothesis that high-mobility group box 1 (HMGB1), a damage-associated molecular pattern (DAMP) with key roles in acute liver injury, may link cell death to injury responses and hepatocarcinogenesis in CLD. While liver-specific HMCB1 deficiency did not significantly affect chronic injury responses such as fibrosis, regeneration, and inflammation, it inhibited ductular/progenitor cell expansion and hepatocyte metaplasia. HMCB1 promoted ductular expansion independently of active secretion in a nonautonomous fashion, consistent with its role as a DAMP. Liver-specific HMCB1 deficiency reduced HCC development in 3 mouse models of chronic injury but not in a model lacking chronic liver injury. As with CLD, HMCB1 ablation reduced the expression of progenitor and oncofetal markers, a key determinant of HCC aggressiveness, in tumors. In summary, HMCB1 links hepatocyte death to ductular reaction, progenitor signature, and hepatocarcinogenesis in CLD.
\end{abstract}

\section{Introduction}

Chronic liver disease (CLD) causes approximately 2 million deaths per year worldwide (1). In patients with CLD, liver cirrhosis and hepatocellular carcinoma (HCC) are the main contributors to morbidity and mortality and remain a significant clinical problem. HCC is the third leading cause of cancer mortality worldwide and the fastest rising cause of cancer mortality in the US $(1,2)$. Importantly, cirrhosis and HCC are strongly linked to CLD and chronic hepatocellular death (3). Accordingly, a large body of welldesigned clinical studies has demonstrated a profoundly increased risk for the development of cirrhosis $(4,5)$ and HCC (6-8) as well as liver-specific mortality (9) in patients with persistently elevated levels of serum alanine aminotransferase (ALT) (reviewed in ref. 3). Interestingly, an increased risk for cirrhosis and HCC development in patients with high ALT is seen in different types of liver diseases, suggesting that cell death is a common risk factor for the progression (3). This concept is further supported by studies in mouse models with genetically induced chronic hepatocyte death, such as mice with hepatocyte-specific deletion of Tak1, Nemo, or Mcl1, which spontaneously develop liver fibrosis and HCC (10-13), suggesting that chronic cell death is sufficient to initiate liver disease development and drive its progression. However, the mechanisms through which hepatocellular death promotes the devel-

Authorship note: $\mathrm{CH}$ and $\mathrm{PH}$ contributed equally to this work. Conflict of interest: The authors have declared that no conflict of interest exists. Submitted: November 17, 2016; Accepted: March 13, 2018. Reference information: J Clin Invest. 2018;128(6):2436-2451. https://doi.org/10.1172/JCI91786. opment of liver cirrhosis and HCC remain poorly understood. Accordingly, there are currently no approved treatments that target cell death pathways to prevent the development of cirrhosis and HCC besides the treatment of the underlying disease.

The liver reacts to hepatocellular death with a wide range of injury responses that include inflammation, hepatocyte regeneration, fibrosis, and the appearance of "ductular reactions." Ductular reactions contain cell populations, termed ductular or hepatic progenitor cells, which are thought to represent an alternative cellular source for the generation of hepatocytes that becomes relevant when hepatocytes lose their capacity to proliferate. While this concept has been developed on the basis of histopathological evidence in patients (14), the role of ductular cells in animal models remains a matter of debate (14). Despite strong expansion of ductular cells, lineage-tracing studies have shown that these cells do not significantly contribute to the generation of functional hepatocytes in the most commonly used models with a "progenitor response," such as 3,5-diethoxycarbonyl-1,4dihydrocollidine (DDC) and choline-deficient, ethionine-supplemented (CDE) diet-fed mice as well as Mdr2-knockout mice, most likely due to the insufficient suppression of hepatocyte proliferation (15-18). On the other hand, mouse and zebrafish models with severely suppressed hepatocyte proliferation have suggested an important role of ductular cells in hepatocyte formation (19-22). Moreover, human LGR5-positive duct-derived liver stem cells differentiate into hepatocytes in vitro and in vivo (23). Furthermore, hepatocytes can undergo reversible ductular metaplasia with expression of A6, CK19, osteopontin (OPN), and Sox9 $(24,25)$. Together, these studies indicate cellular plasticity 
as well as a context-dependent role for specific cell types in the chronically injured liver.

Although hepatic injury responses are geared toward repair and regeneration, they often become maladaptive in the long term, thereby contributing to the development of cirrhosis and HCC (3). In murine models, in which HCC arises in response to carcinogens or in the setting of chronic injury, tumors originate from hepatocytes and not from ductular cells $(26,27)$. In Diethylnitrosamine-induced (DEN-induced) HCC, tumor-forming cells express "progenitor markers" such as A6, CK19, H19, and Epcam (28), suggesting that hepatocyte metaplasia may be a first step in hepatocarcinogenesis. Moreover, a large body of clinical studies from the past decade has shown worse prognosis in HCCs with a "progenitor signature" or expression of oncofetal markers (2936). In summary, there are tight links between the expression of progenitor markers, HCC development, and clinical outcomes.

One of the first events following cell death in the liver and other organs is the release of damage-associated molecular patterns (DAMPs), which mediate sterile inflammation via specific receptors $(37,38)$. In addition to regulating sterile inflammation, it has been suggested that DAMPs may also be involved in repair responses, thereby linking cell death to wound-healing responses such as regeneration and fibrogenesis and possibly also cancer development (38-40). Here, we tested the hypothesis that DAMPs provide a molecular link between chronic liver injury, maladaptive wound healing, and HCC development, focusing on high-mobility group box 1 (HMGB1), a DAMP with key roles in sterile inflammation following acute liver injury (41). Mice with hepatic HMGB1 deficiency displayed a profound reduction of ductular reactions in multiple models of chronic liver injury. Hepatic HMGB1 deficiency reduced hepatocyte metaplasia and inhibited tumor development in 3 HCC models with chronic injury, but not in an HCC model that lacked chronic injury. Moreover, HMGB1 deficiency reduced the expression of progenitor markers, a key feature of aggressive HCC, within tumors. Together, our findings suggest that HMGB1 links hepatocyte injury to ductular reactions, hepatocyte metaplasia, and HCC development in CLD.

\section{Results}

HMGB1 exerts no major impact on hepatic inflammation, regeneration, or fibrogenesis in the chronically injured liver. Previously, we demonstrated a key role for HMGB1 in sterile inflammation following acute liver injury (41). As the majority of morbidity and mortality from liver disease arises in patients with CLD, we now sought to determine whether HMGB1 may also play a role in biological processes that contribute to key features of CLD, such as the induction of inflammation, fibrosis, regeneration, and ductular reactions. As such, it is conceivable that dying hepatocytes might utilize DAMPs to trigger the regeneration of surviving hepatocytes or instruct cells from other compartments to respond to injury and initiate wound-healing responses. To test this hypothesis, we subjected mice with a liver-specific deletion

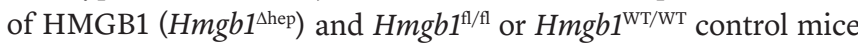
to 3 different, well-characterized models of chronic liver injury, including the DDC diet model (42), $M d r 2$-knockout $\left(M d r 2^{\mathrm{KO}}\right)$ mice (43), and Tak $1^{\text {shep }}$ mice (10). Of note, we had previously shown that $H m g b 1^{\text {shep }}$ mice showed efficient reduction of HMGB1 in paren- chymal cells but did not have any abnormalities in hepatic architecture, injury, fibrogenesis, or gene expression under baseline conditions $(41,44)$, suggesting that intracellular HMGB1 does not play a key role in the maintenance of adult liver homeostasis. $T a k 1^{\mathrm{hhe}}, M d r 2^{\mathrm{KO}}$, and DDC-treated mice had strong inflammation, fibrogenesis, and compensatory proliferation (Supplemental Figure 1-4; supplemental material available online with this article; https://doi.org/10.1172/JCI91786DS1) as well as robust ductular reactions, with characteristic increases in expression of the progenitor markers $C d 133$ and $H 19$ and the oncofetal marker Afp, as well as increased cytokeratin staining (Figure 1, A-F). In contrast to our previous studies of acute liver injury, we only observed a minor to moderate role for HMGB1 in the regulation of neutrophil recruitment, with a significant reduction in the DDC model, a borderline reduction in the $M d r 2^{\mathrm{KO}}$ model, and no significant changes in the Tak1 $1^{\text {thep }}$ model (Supplemental Figure 1, A, C, and E). Likewise, there were no significant differences in $\mathrm{CD} 45^{+}$cell recruitment in any of the 3 CLD models and no consistent alterations of inflammation, with markers such as Tnf and $C d 2 O$ mRNA unaffected and Il6 mRNA upregulated in some models and downregulated in others (Supplemental Figure 1, B, D, and F). We observed no difference in macrophage recruitment in the $M d r 2^{\mathrm{KO}}$ model, a trend toward reduced macrophage numbers in the DDC model, and reduced macrophage numbers in the Tak1 model in HMGB1deleted mice (Supplemental Figure 2). Together, these data suggest that the regulation of sterile inflammation and neutrophil recruitment by HMGB1 is mostly restricted to acute settings, when other inflammatory mediators such as chemokines and cytokines or gut-derived pathogen-associated molecular patterns (PAMPs) are not yet released. We also did not see a major role for HMGB1 in the regulation of liver fibrosis in any of the 3 CLD models (Supplemental Figure 3, A-C). Likewise, we did not observe consistent alterations in proliferation, as determined by Ki67 or phosphorylated histone H3 (p-histone H3) IHC and mKi67 quantitative PCR (qPCR), with similar hepatocyte proliferation in $\mathrm{Hmgbl}^{\mathrm{WT} / \mathrm{WT}}$ and $H m g b 1^{\text {shep }}$ mice in the Tak $1^{\text {thep }}$ model, increased proliferation in $H m g b 1^{\text {shep }}$ mice in the $M d r 2^{\mathrm{KO}}$ model, and decreased proliferation in $H m g b 1^{\text {thep }}$ mice in the DDC diet model (Supplemental Figure $4, \mathrm{~A}-\mathrm{C}$ ). To further determine whether HMGB1 is required for hepatocyte proliferation, we subjected $H m g b 1^{\mathrm{t} / \mathrm{l}}$ and $H m g b 1^{\text {shep }}$ mice to a two-thirds partial hepatectomy (Supplemental Figure $4 \mathrm{D}$ ) or a single injection of $\mathrm{CCl}_{4}$ (Supplemental Figure 4E). We did not observe a role for HMGB1 in the regulation of hepatocyte proliferation in either model, suggesting that the observed minor alterations in liver regeneration may be restricted to some models with ductular reactions.

$H M G B 1$ is required but not sufficient for ductular reactions in the liver. We therefore next examined the possible role of HMGB1 in the regulation of ductular reactions. In contrast to the minor effects on inflammation, proliferation, and fibrosis, we observed a strong and consistent effect of hepatic HMGB1 deficiency on ductular reactions. Hmgb1 $1^{\text {thep }}$ mice that were either crossed with Tak1 $1^{\text {shep }}$ or $M d r 2^{\mathrm{KO}}$ mice or fed a DDC diet, displayed a significant decrease in cytokeratin-positive (Figure 1, A, C, and E) and A6-positive cells (Supplemental Figure 5, A and B), as well as a profound reduction of Cd133, H19, and/or Afp mRNA expression (Figure 1, B, D, and F). Of note, HMGB1 deletion did not affect serum ALT levels (Figure 

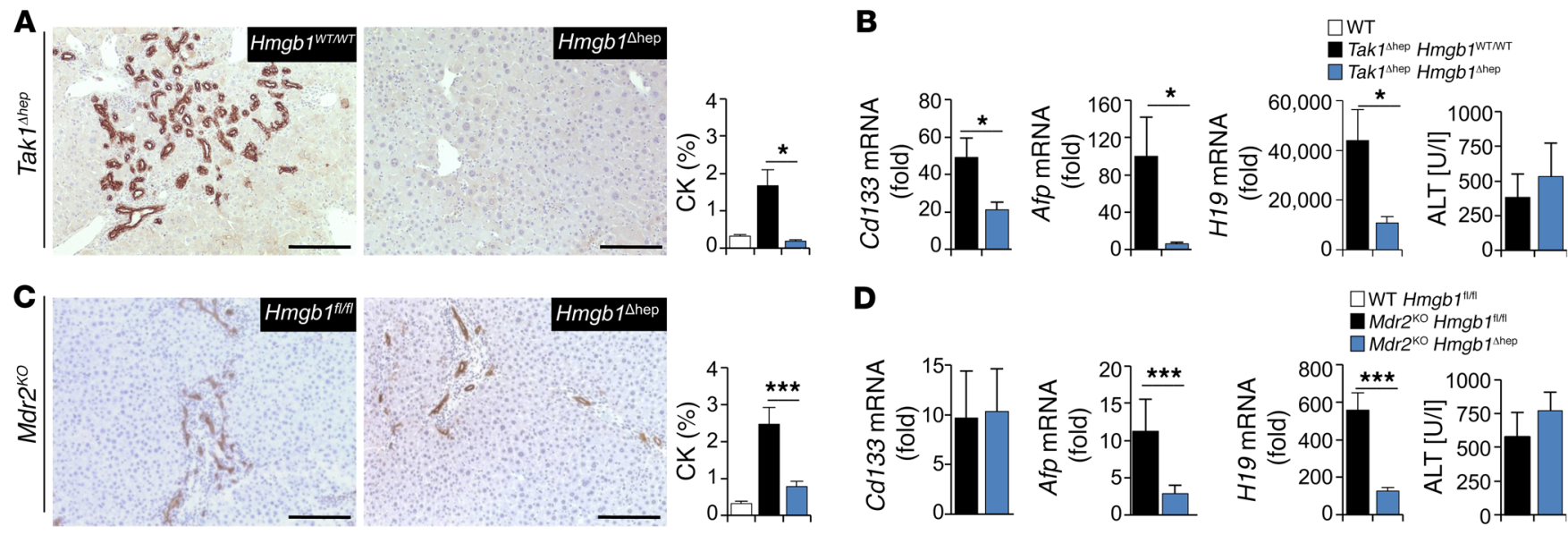

D
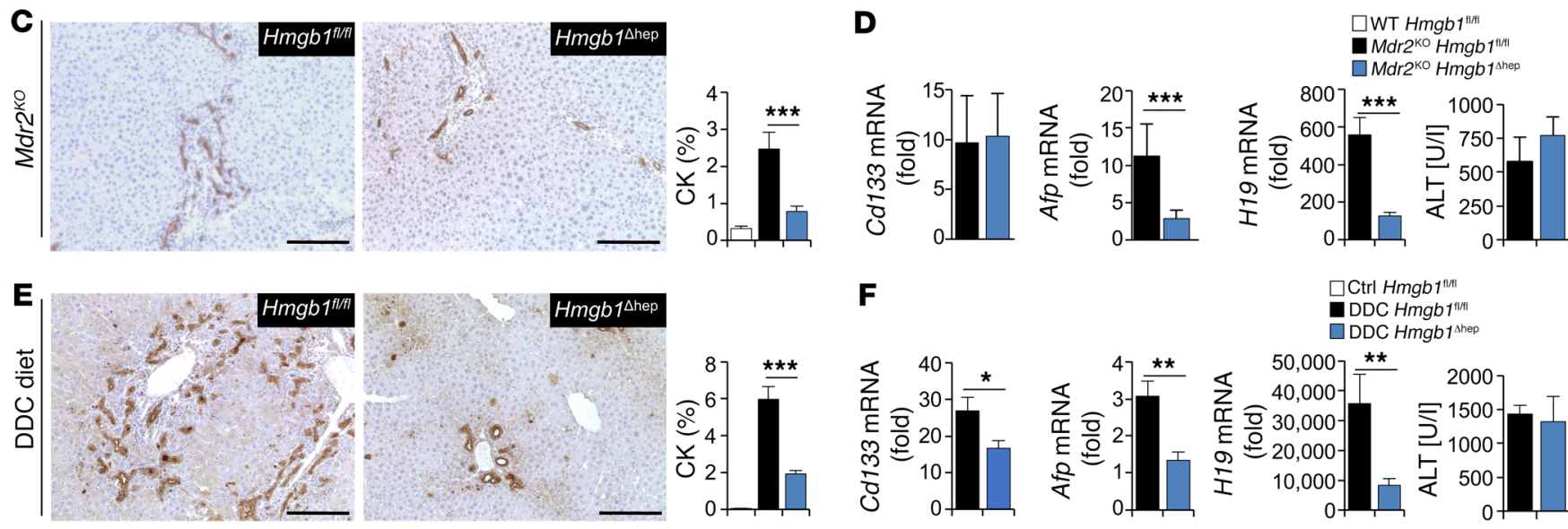

$\mathbf{F}$

$\square$ Ctrl Hmgb1 $1^{\text {ilfil }}$
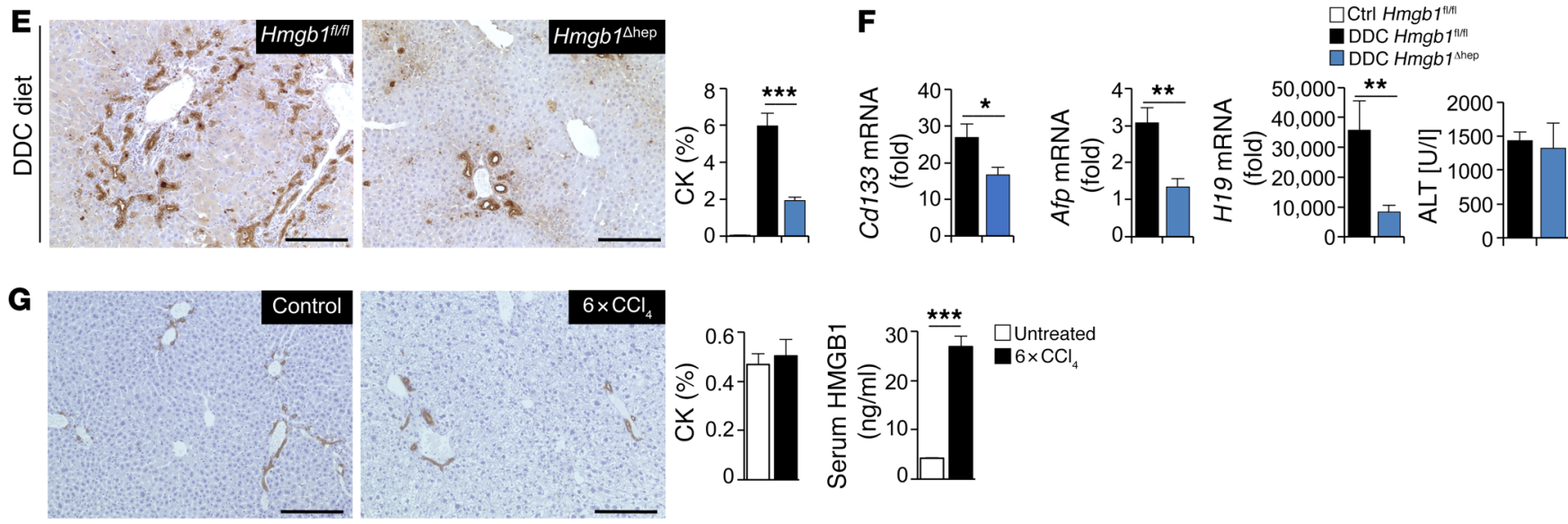

Figure 1. HMGB1 is required for ductular reactions. (A and B) Eight-week-old mice with hepatic deletion of HMGB1 (Hmgb1 $\left.{ }^{\text {Ahep }}, n=9\right)$ had fewer cytokeratin-positive ductular cells (A) and lower expression of Cd133, Afp, and $H 19$ mRNA but similar ALT levels (B) compared with $H m g b 7^{\text {WT/WT }}$ control mice

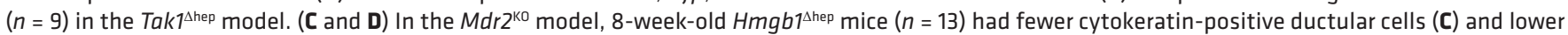
expression of Afp and $H 19$ mRNA but similar ALT levels (E) compared with $H m g b 7^{f l / f l}$ control mice $(n=10)$. (E and F) After 3 weeks on a DDC diet, $H m g b 1^{\text {shep }}$ mice $(n=9)$ had fewer cytokeratin-positive ductular cells $(\mathbf{E})$ and lower expression of Afp and H19 mRNA but similar ALT levels $(\mathbf{F})$ compared with $\mathrm{Hmgb} 7^{f / / f 1}$ control mice $(n=6)$. (C) Untreated mice $(n=4)$ and mice treated with 6 injections of $\mathrm{CCl}_{4}(n=11)$ had similar percentages of cytokeratin-positive cells. HMGB1 serum levels were determined by ELISA in mice that had received $(n=3)$ or not received $(n=2) 6$ injections of CCI. Data are expressed as the mean \pm SEM. qPCR data are shown as the fold induction compared with normal liver. ${ }^{*} P<0.05,{ }^{* *} P<0.01$, and ${ }^{* * *} P<0.001$, by unpaired, 2-tailed $t$ test. Scale bars: $100 \mu \mathrm{m}$. CK, cytokeratin.

1, B, D, and F), thus excluding the possibility that reduced ductular reactions in $\mathrm{Hmgbl}^{\text {shep }}$ mice might have been caused by a decrease in liver injury. Similar to the above models, we also found inhibited ductular reactions in the methione-choline-deficient, ethionine-supplemented (MCDE) diet model (Supplemental Figure 6). Although there was a strong release of HMGB1, as seen by increased HMGB1 serum levels following treatment with the hepatotoxin $\mathrm{CCl}_{4}$, we did not observe ductular reactions in this model (Figure $1 G)$. Together with our findings in the Tak1 ${ }^{\text {thep }}, M d r 2^{\mathrm{KO}}, \mathrm{DDC}$, and MCDE models, our data indicate that HMGB1 is required, but not sufficient, for the development of ductular reactions.

Passively released hepatocyte HMGB1 promotes ductular reactions via cell-extrinsic mechanisms and RAGE. As the deletion strategy via albumin-Cre mice not only ablates HMGB1 in hepatocytes but also in the biliary compartment $(26,41)$, we next sought to exclude the possibility that the lack of HMGB1 in ductular cells impaired their ability to expand. For this purpose, we deleted HMGB1 selectively in hepatocytes using AAV8-TBG-Cre, in which Cre expression is controlled by the hepatocyte-specific thyroxine-binding globulin (TBG) promoter, as a hepatocyte-specific deletion approach $(24,41)$. This strategy resulted in a significant reduction in hepatic Hmgb1 mRNA levels and absent HMGB1 expression in hepatocytes but not in other liver cell types (Figure 2, A and B), thus affecting signals from hepatocytes to other cell types including ductular cells, but leaving HMGB1 within ductular cells intact. $M d r 2^{\mathrm{KO}}$ mice with AAV8-TBG-Cre-mediated hepatocytespecific HMGB1 deletion displayed a significant reduction of the progenitor markers $C d 133$ and $H 19$ and decreased cytokeratin staining in comparison with mice injected with the AAV8-TBGLacZ control virus (Figure 2, C and D). These finding were confirmed in the DDC model, in which we observed a reduction of Cd133 and H19 mRNA levels and cytokeratin staining in mice treated with AAV8-TBG-Cre versus mice that received the AAV8TGB-LacZ virus (Figure 2, E and F). Together, our findings in the $\mathrm{DDC}$ and $M d r 2^{\mathrm{KO}}$ models exclude the possibility that the intracellular loss of HMGB1 within the biliary compartment simply 
A
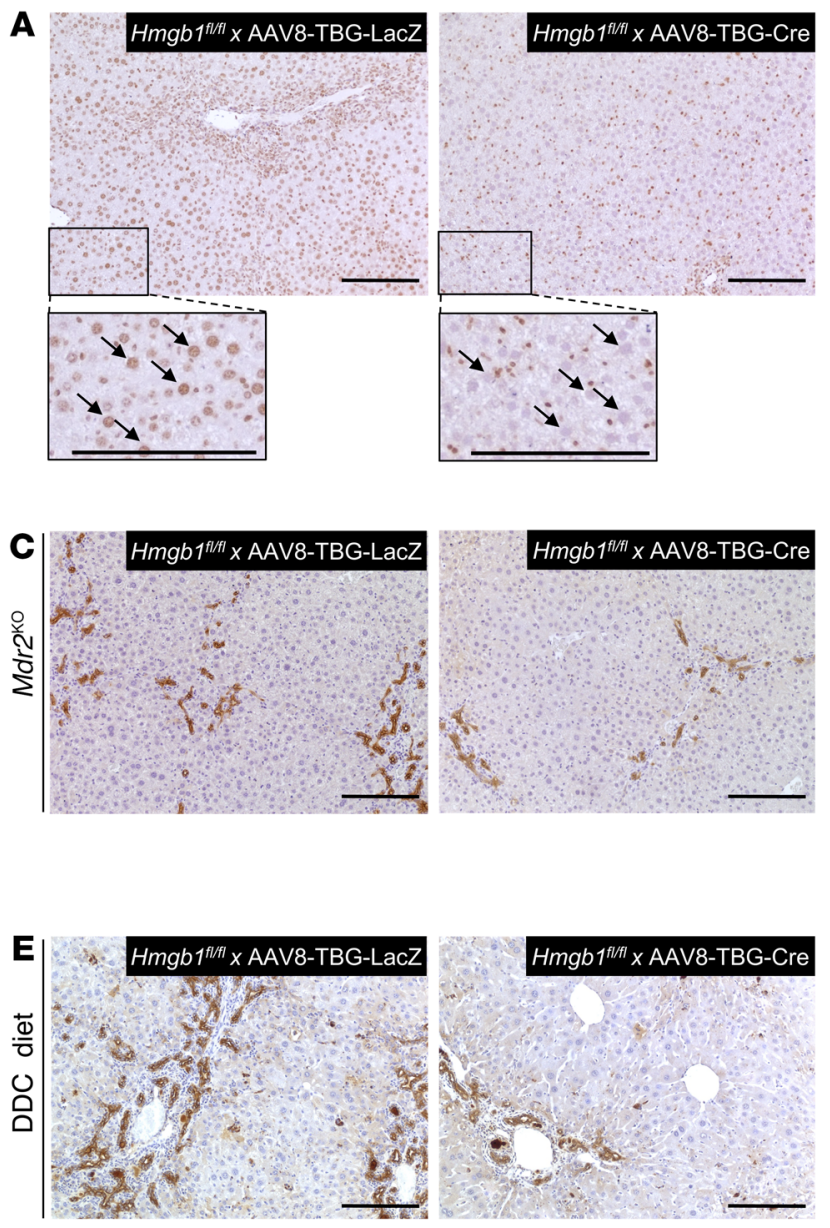

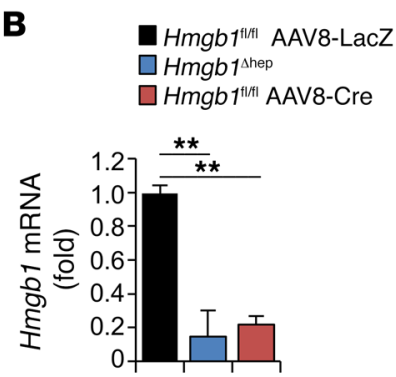

$\square W T H m g b 1^{1 / 1 / 1}$

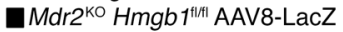

$\square$ Mdr2 ${ }^{\mathrm{kO}} \mathrm{Hmgb}^{1 / 1 / 1}$ AAV8-Cre
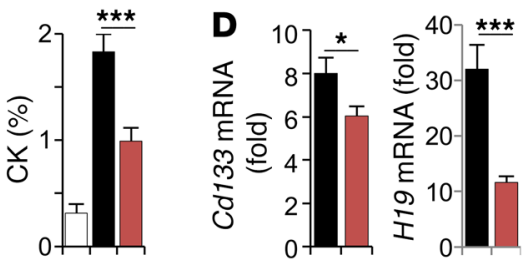

$\square$ Untreated Hmgb1 $1^{1 / 1 / 1}$

DDC Hmgb $1^{\text {thr }}$ AAV8-LacZ

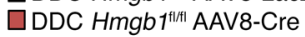
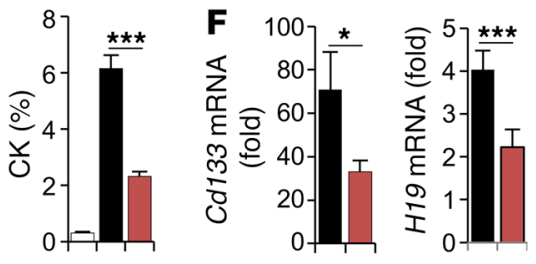

Figure 2. HMGB1 from hepatocellular sources drives ductular reactions. (A) Two-week-old $\mathrm{Hmg} b \mathrm{7}^{\mathrm{fl} / \mathrm{fl}} \mathrm{Mdr2^{ \textrm {kO } }}$ mice were infected with AAV8-TBG-Cre (i.v., $10^{11}$ genome copies, $n=11$ ) or AAV8-TBG-LacZ (i.v., $10^{11}$ genome copies, $n=10$ ) and sacrificed six weeks later. Immunohistochemical HMCB1 staining revealed efficient deletion of HMGB1 from hepatocytes in AAV8-TBG-Cre- but not AAV8-TBG-LacZ-infected mice (arrows indicate hepatocytes). (B) Hmgb1 deletion was confirmed by qPCR in Hmgb $7^{\text {fl/fl }}$ mice infected with AAV8-TBG-Cre or AAV8-TBG-LacZ. (C and D) Immunohistochemical cytokeratin staining (C) and qPCR for $C d 133$ and $H 19$ (D) showed reduced ductular reactions in AAV8-TBG-Cre-infected mice. (E and F) Three-week-old Hmgb7//fli mice were infected with AAV8-TBG-Cre $(n=8)$ or AAV8-TBG-LacZ $(n=7)$ as above, followed by a three-week-long DDC diet, two weeks after AAV infection. Immunohistochemical cytokeratin staining (E) and qPCR for $C d 133$ and $H 19$ (F) revealed reduced ductular reactions in AAV8-TBG-Cre-infected mice. Data are expressed as the mean \pm SEM. qPCR data are shown as the fold induction compared with normal liver. ${ }^{*} P<0.05,{ }^{* *} P<0.01$, and ${ }^{* *} P<0.001$, by unpaired, 2-tailed $t$ test. Scale bars: $100 \mu \mathrm{m}$.

blocked ductular reactions, further supporting our hypothesis that hepatocellular HMGB1, acting as a DAMP, is essential for the induction of ductular reactions.

Extracellular HMGB1 can be released via passive leakage from dead cells (45) or via active secretion, the latter being mediated through acetylation of HMGB1 at multiple lysine residues (46). To determine whether passive release or active secretion of HMGB1 was driving ductular reactions, we inhibited HMGB1 secretion during DDC-induced liver injury using ethyl pyruvate, an established inhibitor of HMGB1 acetylation and secretion (47, 48). Although HMGB1 acetylation was completely suppressed by ethyl pyruvate (Figure 3, A and B), we did not observe significant effects on cytokeratin or A6 expression or on $C d 133$ or $A f p$ mRNA levels and only a borderline significant reduction of $H 19$ mRNA (Figure 3, C-E). Moreover, HMGB1 serum levels were not significantly decreased (Figure 3F), despite the observed loss of acetylated HMGB1. Together, these findings suggest that pas- sive HMGB1 release from dying hepatocytes, rather than active secretion, is the dominant driver of ductular reactions in the settings we investigated.

HMGB1 binds to several receptors including RAGE (encoded by Ager), TLR4, and TLR9. We next determined which one of these three HMGB1 receptors was involved in promoting ductular reactions in vivo, using Ager-deficient (Rage $\left.{ }^{\mathrm{KO}}\right)$, Tlr4-deficient $\left(T l r 4^{\mathrm{KO}}\right)$, and Tlr9-deficient $\left(T \operatorname{lr} 9^{\mathrm{KO}}\right)$ mice. We found strongly reduced ductular reactions with significantly decreased cytokeratin staining and reduced $H 19$ and Afp mRNA expression, as well as a trend toward decreased Cd133 mRNA levels in livers from Rage ${ }^{\mathrm{KO}}$ mice (Figure $\left.4 \mathrm{~A}\right)$. In contrast, $T l r 4^{\mathrm{KO}}$ and $\operatorname{Tl} 9^{\mathrm{KO}}$ mice did not show impaired ductular reactions in the DDC diet model, as demonstrated by unaltered cytokeratin staining and unaltered or increased Cd133, H19, and Afp mRNA expression in Tlr $4^{\mathrm{KO}}$ and $T l r 9^{\mathrm{KO}}$ mice (Figure 4, B and C). As with $H m g b 1^{\text {shep }}$ mice, we did not find alterations in liver injury in Rage ${ }^{\mathrm{KO}}$ mice, thus excluding 
A

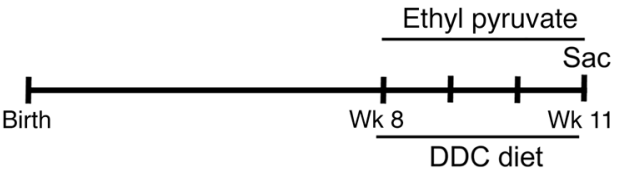

B

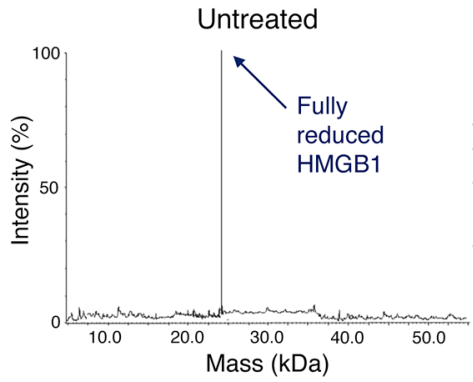

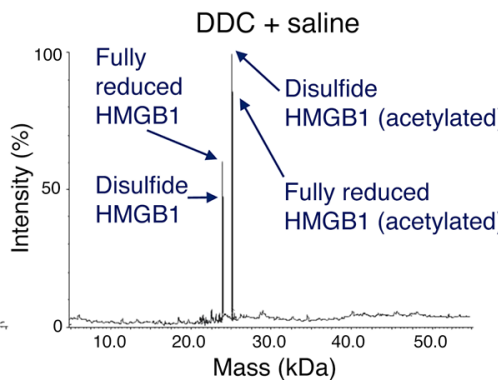

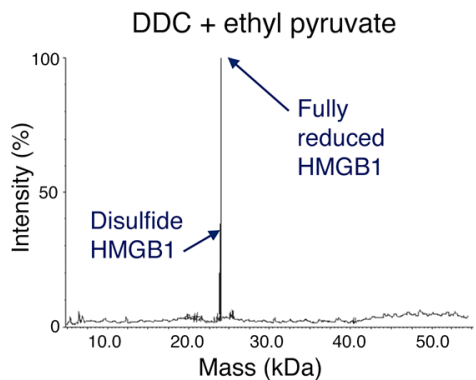

C

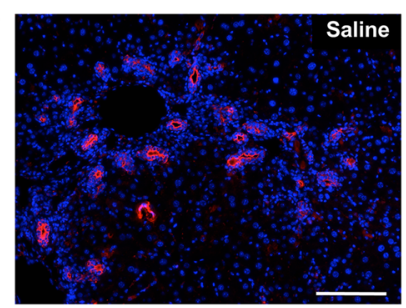

D

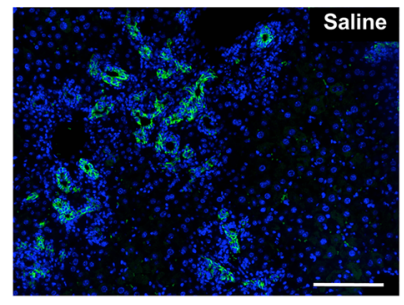

E

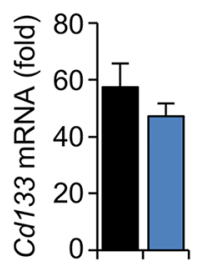

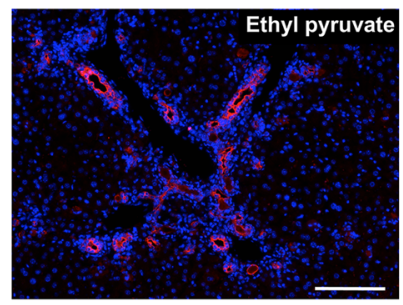
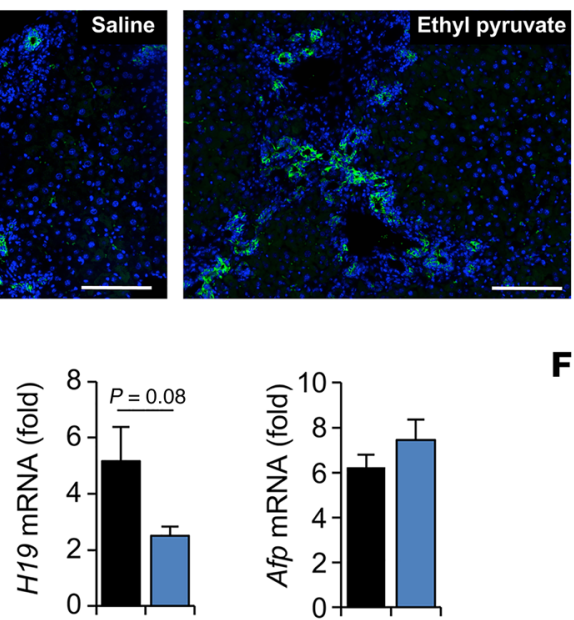

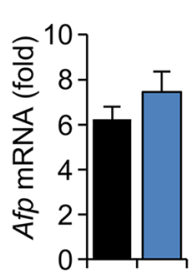

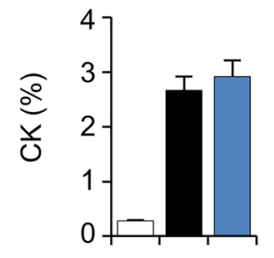

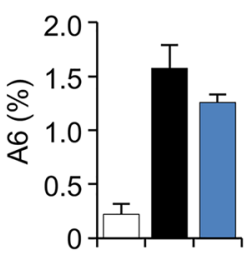

$\mathbf{F}$

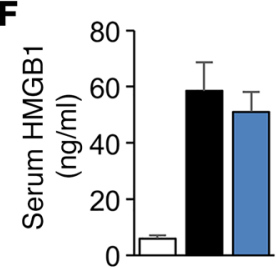

$\square$ Control

DDC + saline

$\square$ DDC + ethyl pyruvate
Figure 3. Active HMGB1 secretion does not drive ductular reactions. (A) $\mathrm{C} 57 \mathrm{BL} / 6$ male mice were pretreated with saline $(n=11)$ or ethyl pyruvate $(n=8)$, followed by a DDC diet for 3 weeks. (B) HMCB1 isoforms were determined by LC-MS/MS in plasma from untreated mice as well as in plasma from DDC diet-fed mice that received saline or ethyl pyruvate. (C and D) IHC showed similar expression levels of cytokeratin (C) and A6 (D) in saline- and ethyl pyruvate-treated mice. (E) qPCR showed similar expression levels of Cd133, $A f p$, and $H 19$ mRNA in mice treated with saline or ethyl pyruvate. (F) Serum HMGB1 levels in control and DDC-treated mice were determined by ELISA. Data are expressed as the mean \pm SEM qPCR data are expressed as the fold induction compared with normal liver. Statistical significance was determined by unpaired, 2-tailed $t$ test. Scale bars: $100 \mu \mathrm{m}$. the possibility that reduced ductular reactions were merely a consequence of less injury in the Rage $\mathrm{e}^{\mathrm{KO}}$ mice (Figure $4 \mathrm{~A}$ ).

Since we also observed small decreases in macrophages in HMGB1-deleted mice in some models (Supplemental Figure 2), we additionally sought to determine whether macrophages might be a cell population through which HMGB1 indirectly triggers progenitor responses. However, consistent with previous studies (49), we observed no decrease in ductular reactions following macrophage depletion (Supplemental Figure 7). Given these findings, we subsequently focused on the direct effect of HMGB1 on bipotential progenitor cells, with the goal of identifying the signals through which HMGB1 affects ductular reactions.

Disulfide HMGB1 mediates effects on progenitor cells via Erk. HMGB1 bioactivity is dependent on posttranslational modifications, with fully reduced HMGB1 promoting cell migration but not inflammation, whereas disulfide HMGB1 has cytokine-like proinflammatory activity (50). Similar to the findings from our previ- ous studies on acute liver injury, we found that disulfide HMGB1 was the most abundant form of HMGB1 in the chronically injured liver and that this form increased with the duration of liver injury (Figure 5A). More important, stimulation with these 2 forms of HMGB1, which have distinct bioactivities, revealed that only the disulfide form of HMGB1 upregulated Cd133 mRNA in bipotential murine oval liver (BMOL) cells (Figure 5B), a well-characterized bipotential liver progenitor cell line (51). As YAP and Notch are well-established and powerful signals driving hepatocyte metaplasia toward a ductular phenotype in adult livers $(24,52)$ as well as hepatocarcinogenesis (53), we determined whether HMGB1 could act through these pathways. However, we found no difference in Notch target gene expression after treating BMOL cells with HMGB1, which was further confirmed by similar levels of Notch target genes in control and HMGB1-deleted mice in vivo in multiple models (Supplemental Figure 8). Following treatment of BMOL cells with HMGB1, we found that YAP target genes as 
A

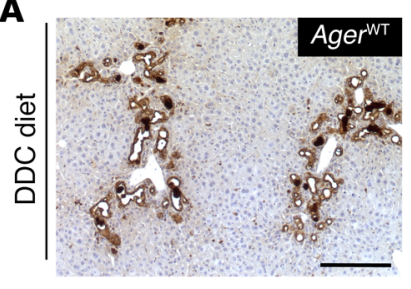

B

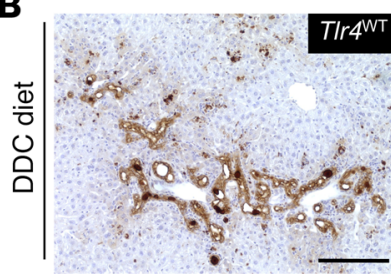

TIr4WT

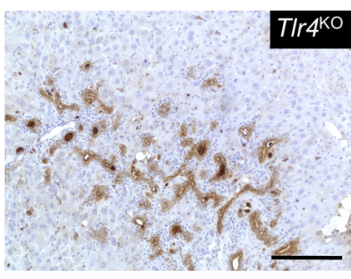

$\operatorname{TIr} 4^{\mathrm{KO}}$

TIrgKO

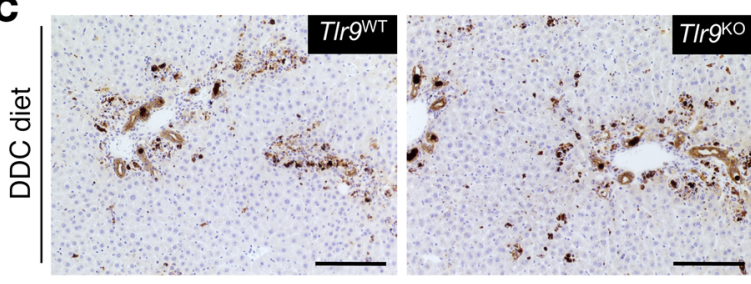

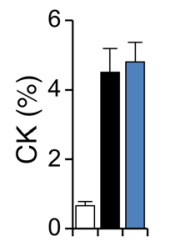
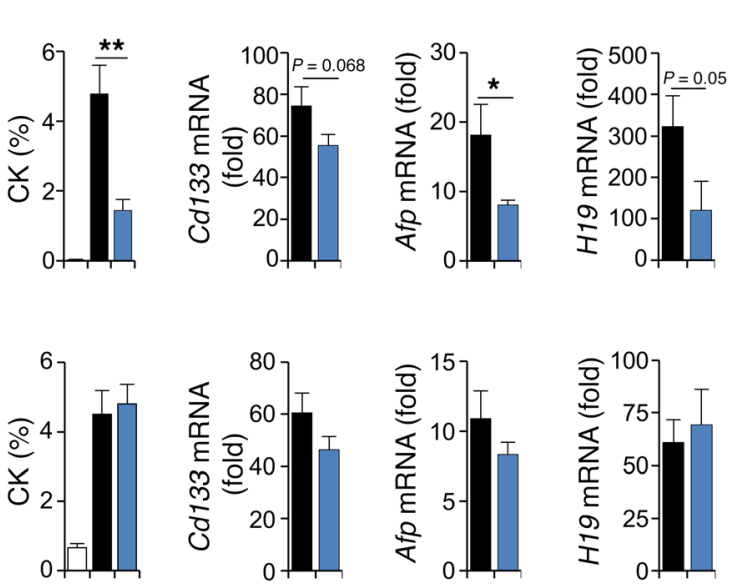

$\square$ Control WT

DDC WT

$\square \mathrm{DDC}$ Ager ${ }^{\mathrm{Ko}}$

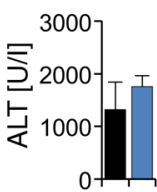

$\square$ Control WT

-DDC WT

$\square$ DDC TIr4 ${ }^{\mathrm{KO}}$

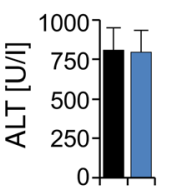
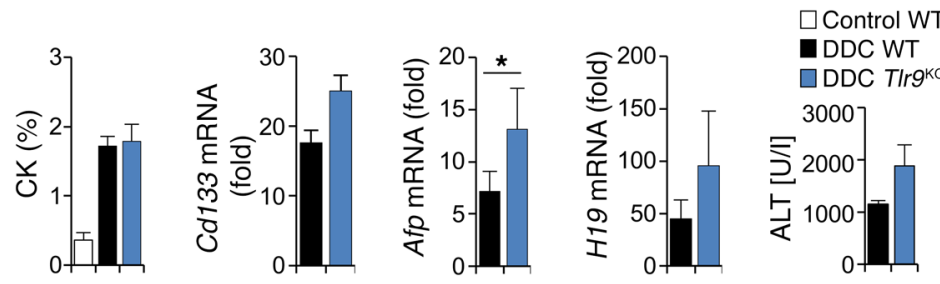

Figure 4. HMGB1 drives ductular reactions via RAGE but not TLR4 or TLR9. Male mice were fed a DDC diet for 3 weeks. (A) Cytokeratin expression and Cd133, Afp, and H19 mRNA levels were determined by IHC and qPCR in WT ( $n=7)$ and Rage ${ }^{\mathrm{KO}}(n=8)$ mice. Liver injury was assessed by serum ALT levels. (B) Cytokeratin expression, Cd133, Afp, and H19 mRNA expression, and liver injury were determined in WT ( $n=7)$ and T/r4 ${ }^{\mathrm{Ko}}(n=8)$ mice as above. (C) Cytokeratin expression, Cd133, Afp, and H19 mRNA expression and liver injury were determined in WT $(n=9)$ and Tlrgko $(n=10)$ mice. Data are expressed as the mean \pm SEM. qPCR data are shown as the fold induction compared with normal liver. ${ }^{*} P<0.05$ and ${ }^{* *} P<0.01$, by unpaired, 2 -tailed $t$ test. Scale bar: $100 \mu \mathrm{m}$.

well as YAP reporter activity decreased (Supplemental Figure 9). In contrast, we observed a decrease in some YAP target genes in HMGB1-deleted mice in vivo (Supplemental Figure 9). As our experiment had not shown any direct effect of HMGB1 on YAP target genes in BMOL progenitor cells in vitro, and as we did not see a major role for hepatocyte YAP in ductular reactions after 3 weeks of DDC diet feeding (data not shown), we reasoned that the reduction of YAP target gene expression in HMGB1-deleted mice probably reflects the decrease in ductular cells (which are enriched in YAP and YAP target genes) rather than indicating a lack of HMGB1-induced YAP activation. Accordingly, when normalizing our qPCR results to the progenitor marker $C d 133$, the YAP target gene Ctgf was significantly $(P<0.05)$ upregulated in HMGB1deleted mice in the DDC model, and significantly $(P<0.05)$ downregulated in the Tak1 $\Delta$ hep model where all others showed no significant differences between floxed and HMGB1-deleted mice (data not shown). Therefore, we sought to identify additional signals through which disulfide HMGB1 may affect ductular reactions. For this purpose, we performed a phospho-screen in BMOL cells treated with disulfide HMGB1. In this screen, we found that Erk phosphorylation and phosphorylation of its target CREB, but not other pathways, were strongly induced by disulfide HMGB1 (Figure 5C), which was further confirmed by Western blot analysis (Figure 5D, top). In contrast, fully reduced HMGB1 did not stimulate Erk phosphorylation (Figure 5D, bottom). Inhibition of Erk phosphorylation strongly decreased the expression of $C d 133$ mRNA in BMOL cells (Figure 5E), suggesting that this pathway mediates the effects of HMGB1 on progenitor cells. This finding is supported by previous studies that have described a key role of Erk in the regulation of $\operatorname{CD} 133(54,55)$. Moreover, treatment with disulfide HMGB1 moderately but significantly promoted the proliferation of BMOL cells (Figure 5F), suggesting that HMGB1 contributes to progenitor expansion via this pathway. Indeed, we found that there was a reduction of p-histone H3 (Ser10)/Krt19double-positive proliferating progenitor cells in HMGB1-deleted mice after 3 weeks of a DDC diet (Figure 5G). Consistent with our in vivo studies in knockout mice as well as a previously published study (56), we also found that inhibition of RAGE blunted HMGB1-induced Erk phosphorylation, whereas TLR4 or TLR9 blockade had no major effect (Figure $5 \mathrm{H}$ ). In summary, our findings suggest that HMGB1-induced activation of RAGE triggers the proliferation and expansion of progenitor cells.

HMGB1 promotes hepatocyte metaplasia and links chronic injury to HCC development. Chronic liver injury may result in the development of HCC, the third leading cause of cancer mortality worldwide. Accordingly, chronic hepatocellular death strongly increases the risk for HCC development $(6,7)$. Moreover, the expression of progenitor and oncofetal genes is common in HCC and adversely affects prognosis (29-36). Consistent with previous studies $(24,25)$, we found via lineage tracing with AAV8-TBG-Cre that hepatocytes undergo ductular metaplasia, as seen by coexpression of the Cre reporter TdTom, demonstrating hepatocyte origin, and ductular markers A6, OPN, and Sox9 (Figure 6, A-D). In mice that had been fed a DDC diet for 3 weeks, A6-positive 
A
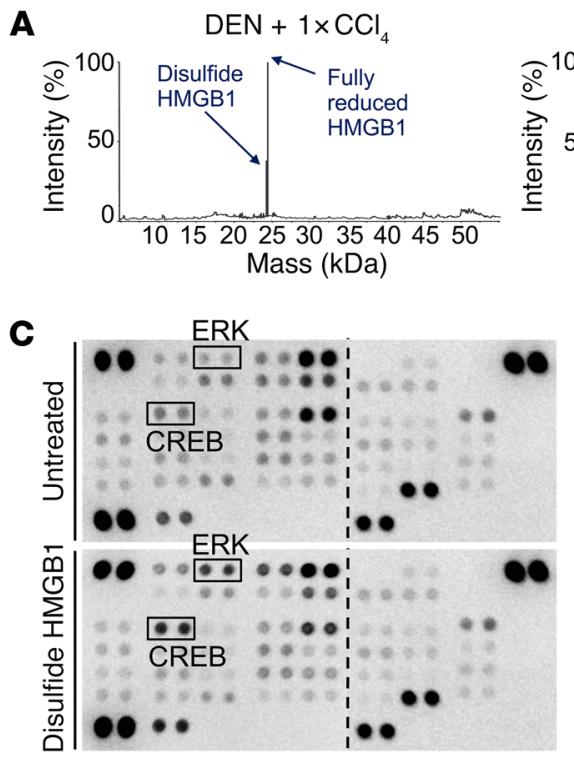

E

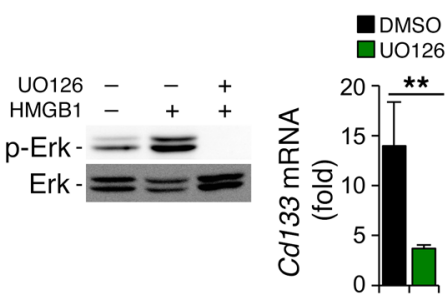

$\mathrm{DEN}+6 \times \mathrm{CCl}_{4}$

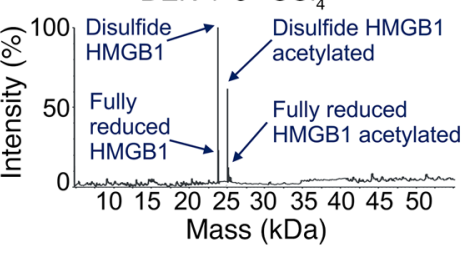

B

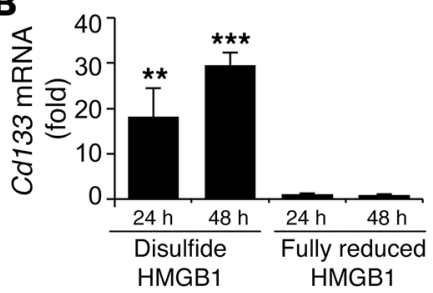

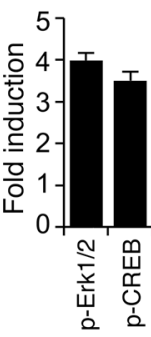
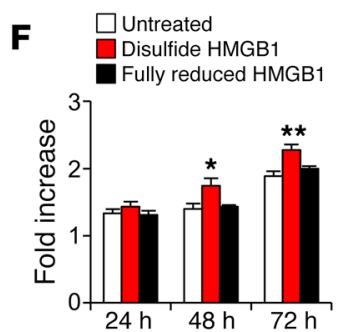

H

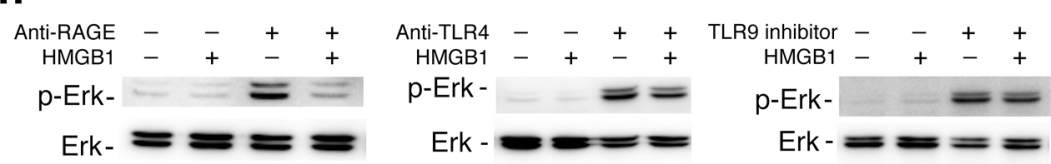

G

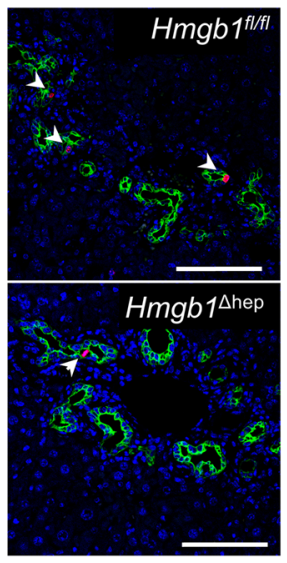

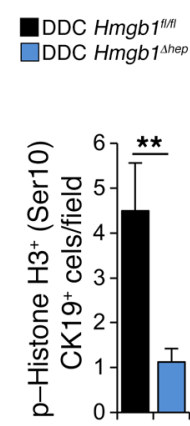

Figure 5. Disulfide HMGB1, but not fully reduced HMGB1, upregulates CD133 and promotes progenitor/ductular proliferation. (A) HMCB1 isoforms were determined by LC-MS/MS in plasma from mice treated with DEN plus 1 injection of $\mathrm{CCl}_{4}$ or with DEN plus 6 injections of $\mathrm{CCl}$. (B) BMOL cells were treated with recombinant disulfide HMGB1 $(2 \mu \mathrm{g} / \mathrm{ml})$ and fully reduced HMGB1 $(2 \mu \mathrm{g} / \mathrm{ml})$ for the indicated durations, followed by analysis of Cd133 mRNA by qPCR. (C) Phospho-kinase screen in untreated and disulfide HMCB1-treated $(0.5 \mu \mathrm{g} / \mathrm{ml}$ for 30 minutes) BMOL cells. (D) BMOL cells were treated with disulfide HMCB1 $(0.5 \mu \mathrm{g} / \mathrm{ml})$ and fully reduced HMCB1 $(0.5 \mu \mathrm{g} / \mathrm{ml})$ for the indicated durations, followed by Western blot analysis for phosphorylated and total Erk. (E) BMOL cells were pretreated with the MEK inhibitor U0126 (10 mM) for 30 minutes, followed by treatment with disulfide HMCB1 (0.5 $\mu \mathrm{g} / \mathrm{ml})$ and qPCR analysis of Cd133 mRNA. (F) Proliferation was determined by MTT assay in BMOL cells treated with disulfide HMGB1 ( $0.5 \mu \mathrm{g} / \mathrm{ml})$ or fully reduced HMCB1 $(0.5 \mu \mathrm{g} / \mathrm{ml})$ for the indicated durations. (C) Cytokeratin 19 and $\mathrm{p}$-histone $\mathrm{H3}$ (Ser10) double-positive cells were detected by confocal microscopy in $\mathrm{Hmg} \mathrm{f}^{\mathrm{fl} / \mathrm{fl}}$ (black bar) and Hmgb $1^{\text {shep }}$ (blue bar) mice fed a DDC diet ( $n=6$ and $n=9$ mice, respectively), and then cells were quantified. (H) BMOL cells were preincubated with RAGE- and TLR4-blocking antibodies, TLR9 inhibitor, or the appropriate controls, followed by treatment with disulfide HMGB1 (0.05 $\mu \mathrm{g} / \mathrm{ml})$ for 15 minutes and p-Erk and Erk Western blot analysis. Data are expressed as the mean \pm SEM. qPCR data are expressed as the fold induction compared with untreated cells, and proliferation is expressed as the fold increase compared with the 0 -hour time point. ${ }^{*} P<0.05$, ${ }^{* *} P<0.01$, and ${ }^{* * *} P<0.001$, by unpaired, 2-tailed $t$ test. Scale bars: $100 \mu \mathrm{m}$.

hepatocytes constituted less than $10 \%$ of all A6-positive cells in the liver (Figure 6B). Of note, A6-positive metaplastic hepatocytes were reduced by $68 \%(P<0.001)$ in HMGB1-deleted mice (Figure 6B). Likewise, we also detected a significant reduction of TdTomand OPN-double-positive and TdTom- and Sox9-double-positive hepatocytes (Figure 6, C and D) as well as a significant reduction of $S p p 1$ (encoding OPN) and Sox 9 mRNA levels (Figure 6, E and F) in HMGB1-deleted mice. Together, these findings suggest that hepatocellular HMGB1 promotes ductular metaplasia of hepatocytes, but that this largely HMGB1-dependent response contributed only a small fraction to the overall ductular response at the time points that we studied. As A6-, CK19-, AFP-, H19-, Epcam- and Sox9expressing cells may function as liver cancer progenitors (28), and given that HMGB1 had a major role in regulating the expression of these markers in the liver as well as the expression of A6 in hepatocytes in particular, we next tested the hypothesis that HMGB1 may provide a link between hepatocellular death and HCC development. For this purpose, we subjected $H m g b 1^{\mathrm{A} / \mathrm{fl}}$ and $H m g b 1^{\text {shep }}$ mice to models of hepatocarcinogenesis that either incorporated or lacked chronic liver injury (Figure 7, A-G). To mimic the development of HCC in chronically injured, inflamed, and fibrotic livers in patients, we used the well-established combination of the 
A
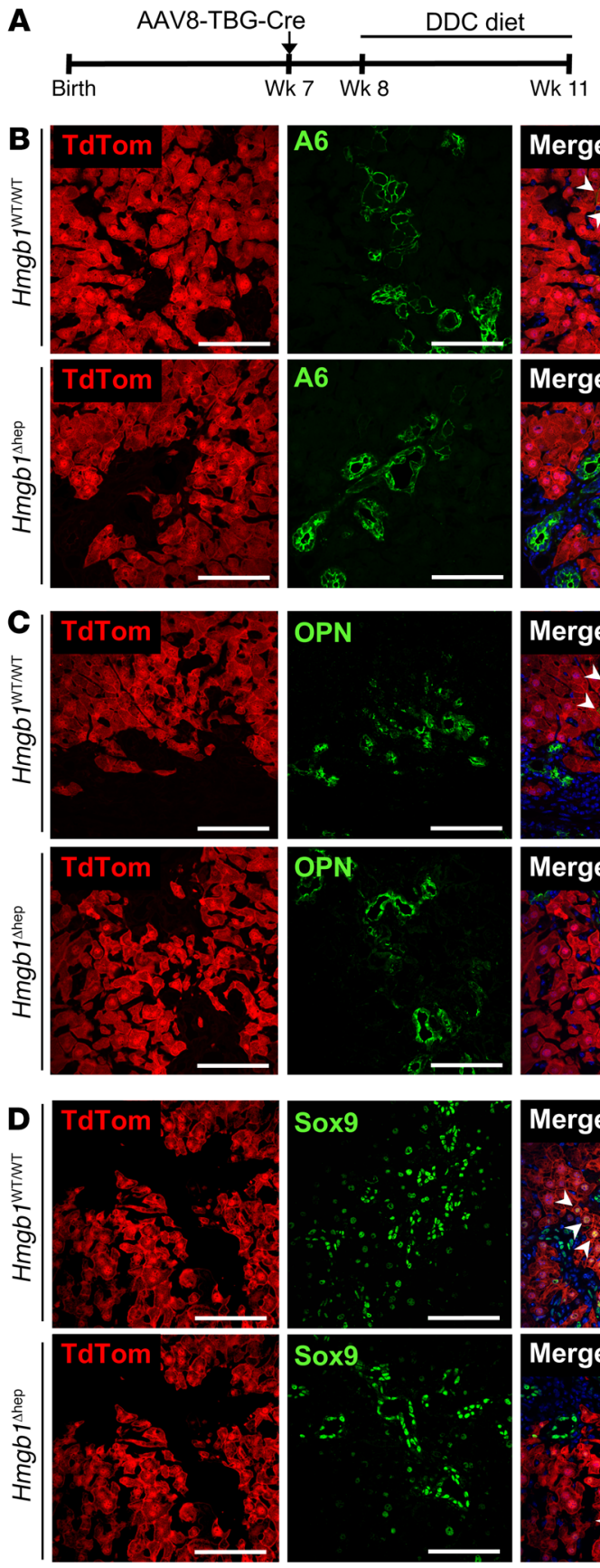
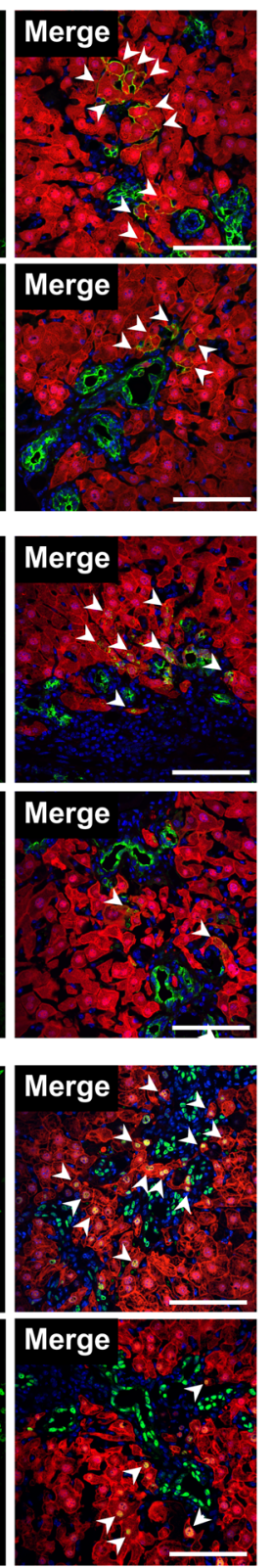

DDDC Hmgb1 1wTwT $\square$ DDC Hmgb1 $1^{\text {shep }}$

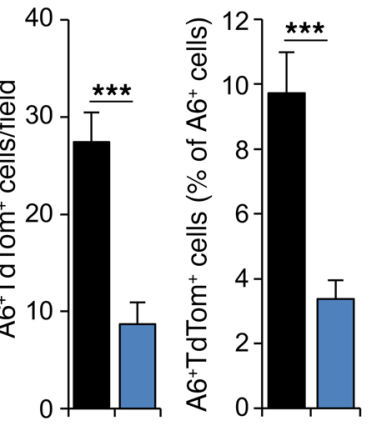

E
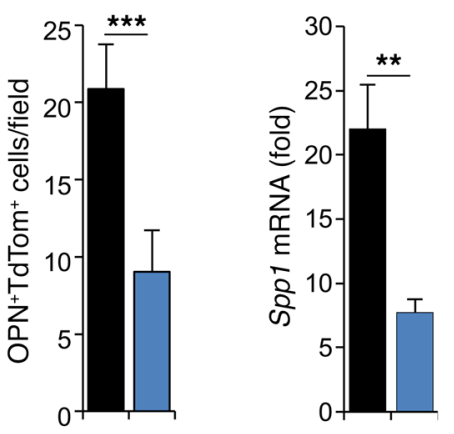

$\mathbf{F}$

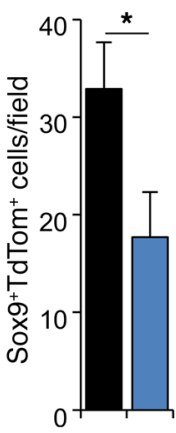

Figure 6. HMGB1 promotes ductular metaplasia of hepatocytes. (A) Seven-week-old HMCB1 WT mice (Hmgb7 ${ }^{\mathrm{WT} / \mathrm{WT}}, n=12$ ) or mice with floxed HMCB1 alleles ( $\left.\mathrm{Hmgb}^{\text {thep }}, n=8\right)$, both expressing TdTom, were infected with AAV8-TBG-Cre (i.v., $10^{11}$ genome copies), followed by a three-week-long DDC diet, one week after AAV infection (B-D) Hepatocyte metaplasia was compared using dual immunofluorescence staining for $A 6$ and TdTom (B), OPN and TdTom (C), or Sox9 and TdTom (D). $\mathrm{Hmgb}^{\text {shep }}$ mice showed a reduction in cells double-positive for $A 6$ and TdTom compared with $\mathrm{Hmg} b 7^{\text {WT/WT }}$ mice as well as of the percentage of A6- and TdTom-double-positive cells among all A6-positive cells (B). $\mathrm{Hmgb}^{\text {thep }}$ mice showed a reduction of OPN- and TdTom-double-positive cells (C) as well as a reduction of Sox9and TdTom-double-positive cells (D) when compared with $\mathrm{Hmg}_{\mathrm{m}}$ 1 $^{\mathrm{WT} / \mathrm{WT}}$ mice. (E and F) qPCR showed decreased expression of OPN (encoded by Spp1) and Sox9 mRNA in DDC diet-treated $\mathrm{Hmg}_{\mathrm{m}} 1^{\text {hep }}$ mice. qPCR data are shown as the fold induction compared with normal liver. Data are expressed as the mean \pm SEM. ${ }^{*} P<0.05,{ }^{* *} P<0.01$, and ${ }^{* *} P<0.001$, by unpaired, 2-tailed $t$ test. Scale bar: $100 \mu \mathrm{m}$. carcinogen DEN with chronic injection of the hepatotoxin $\mathrm{CCl}_{4}$ or with the above-described DDC diet model $(57,58)$. The DEN plus $\mathrm{CCl}_{4}$ model resulted in a significantly increased release of HMGB1 in comparison with the DEN-only model (Figure 7D). In the DEN plus $\mathrm{CCl}_{4}$ model, we found a significant reduction of HCC development, as determined by tumor numbers and liver/body weight ratios, and a borderline significant reduction $(P=0.06)$ in tumor size (Figure 7, A-C). To further confirm the contribution of HMGB1 to injury-driven hepatocarcinogenesis, we next tested its role in mice with hepatocyte-specific deletion of Tak1. In this model, mice spontaneously develop HCC $(10,59)$ as a result of chronic cell death, without the need for injection of carcinogens. As with the DEN plus $\mathrm{CCl}_{4}$ model, we observed a significant reduction in tumor numbers and size and a borderline significant reduction in liver/body weight ratios (Supplemental Figure 10, A-C). Likewise, we also observed a reduction in tumor formation in mice treated with DEN plus a DDC diet (Supplemental Figure 10, D and E), which triggers the development of HCC in the presence of chronic injury, ductular reactions, and progenitor marker expression (58). In contrast, when we subjected $H m g b 1^{\text {shep }}$ or $H m g b 1^{\mathrm{l} / \mathrm{fl}}$ control mice to DEN-induced hepatocarcinogenesis, a purely genotoxic model without chronic liver injury or strong HMGB1 release (Figure 7D), we observed abundant tumors in both groups of mice, without significant differences in tumor number, size, or liver/body weight ratio (Figure 7, $E-G$ ). The finding that cell death was required to reveal effects of hepatic HMGB1 strongly suggests that HMGB1 
A

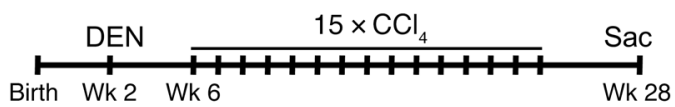

B
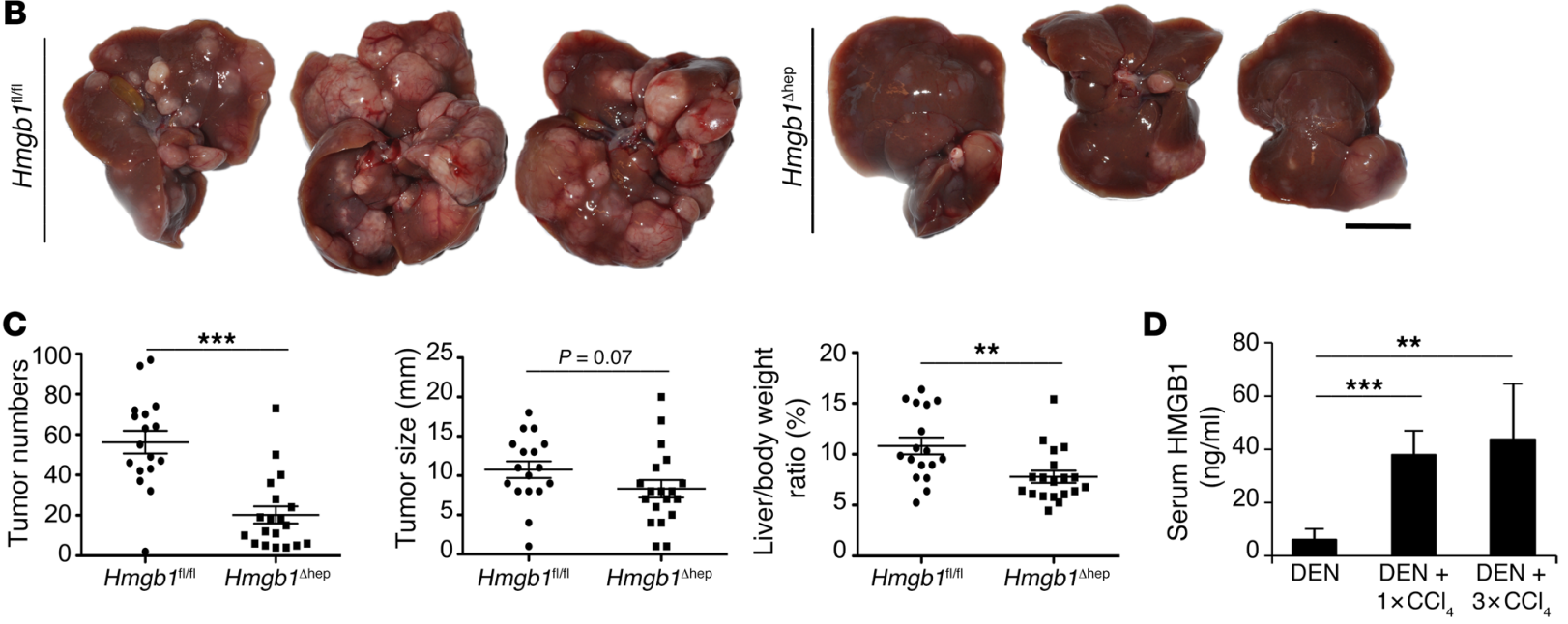

$\mathbf{E}$
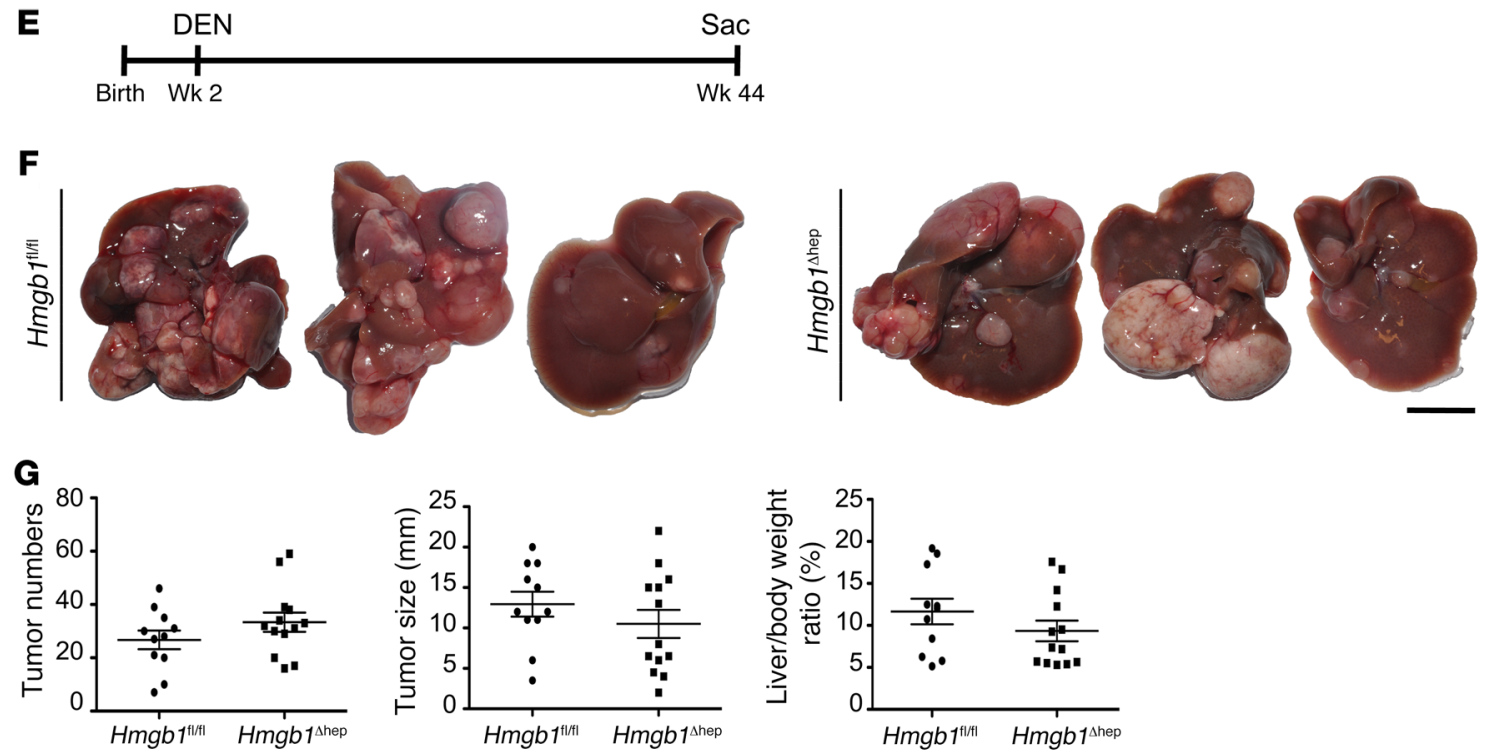

Figure 7. HMCB1 promotes hepatocarcinogenesis in the presence but not the absence of chronic liver injury. (A-C) Male $H m g b 7^{\text {fl/fl }}(n=17)$ and $H m g b 1^{\text {thep }}$ $(n=19)$ mice were treated with DEN and $\mathrm{CCl}_{4}$ and sacrificed at the indicated time point (A). Livers were photographed (B), and tumor numbers, size, and liver/body weight ratios were determined (C). (D) HMGB1 serum levels were determined by ELISA in mice that had either received DEN only $(n=4)$, DEN

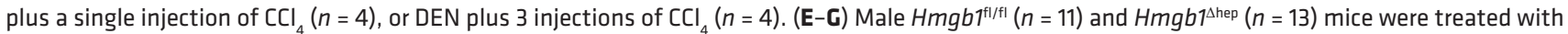
DEN and sacrificed at week 44 (E). Livers were photographed (F) and tumor numbers, size, and liver/body weight ratios were determined (C). Data are expressed as the mean \pm SEM. ${ }^{* *} P<0.01$ and ${ }^{* *} P<0.001$, by unpaired, 2 -tailed $t$ test. Scale bars: $1 \mathrm{~cm}$.

acted as a DAMP and excludes the possibility that the lack of intracellular HMGB1 might have simply impaired the ability of tumor-initiating cells to form tumors.

HMGB1 controls the progenitor signature but not fibrosis, inflammation, or proliferation in HCC. To better understand the mechanisms through which HMGB1 promotes hepatocarcinogenesis in the injured liver, we focused on tumor-promoting injury responses. Similar to our findings in chronic injury models, we did not observe differences in proliferation, fibrosis, or inflammation, as demonstrated by similar levels of Ki67 ${ }^{+}$, Picrosirius red, $\mathrm{CD} 45^{+}$, and $\mathrm{F} 4 / 80^{+}$staining, as well as similar levels of inflammatory gene expression in tumors from $H m g b 1^{\text {hep }}$ and $H m g b 1^{\mathrm{I} / \mathrm{fl}}$ mice (Figure 8, A-C, and Supplemental Figure 11, A-C). Likewise, we did not find a role for HMGB1 in the recruitment of neutrophils (Figure $8 \mathrm{D})$, a cell population that contributes to the development of liver cancer (60) and whose numbers positively correlate with worse prognosis in HCC $(61,62)$. Given that we observed a moderate role for HMGB1 in neutrophil recruitment in earlier stages of injury (Supplemental Figure 1, A-C), and since HMGB1 might not only contribute to neutrophil recruitment but also to neutrophil activation, we additionally performed functional experiments with mice deficient for neutrophil elastase (encoded by Elane), which 
A

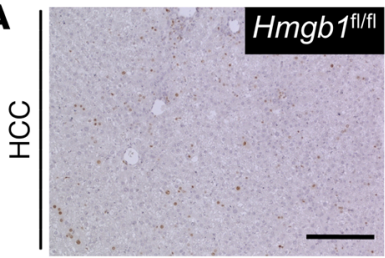

B
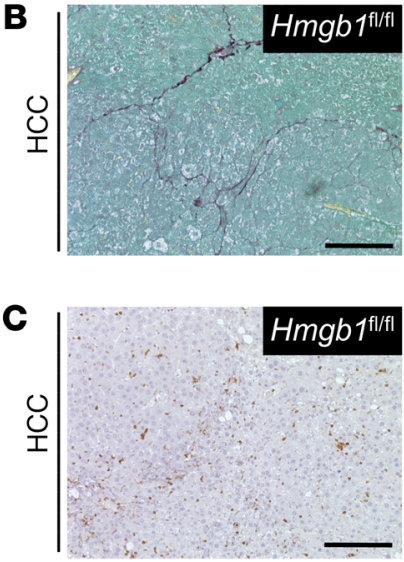

D

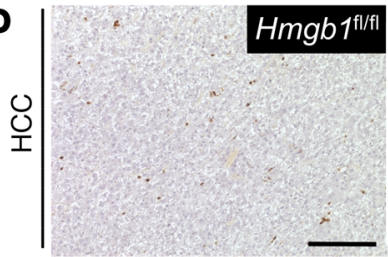

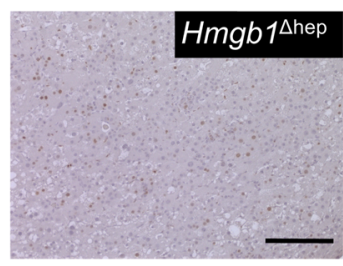
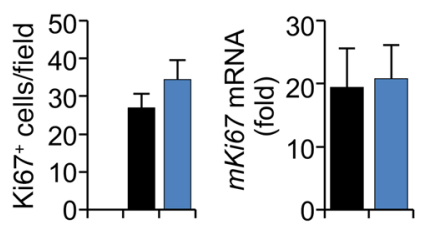

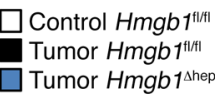

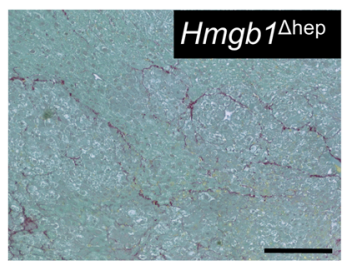
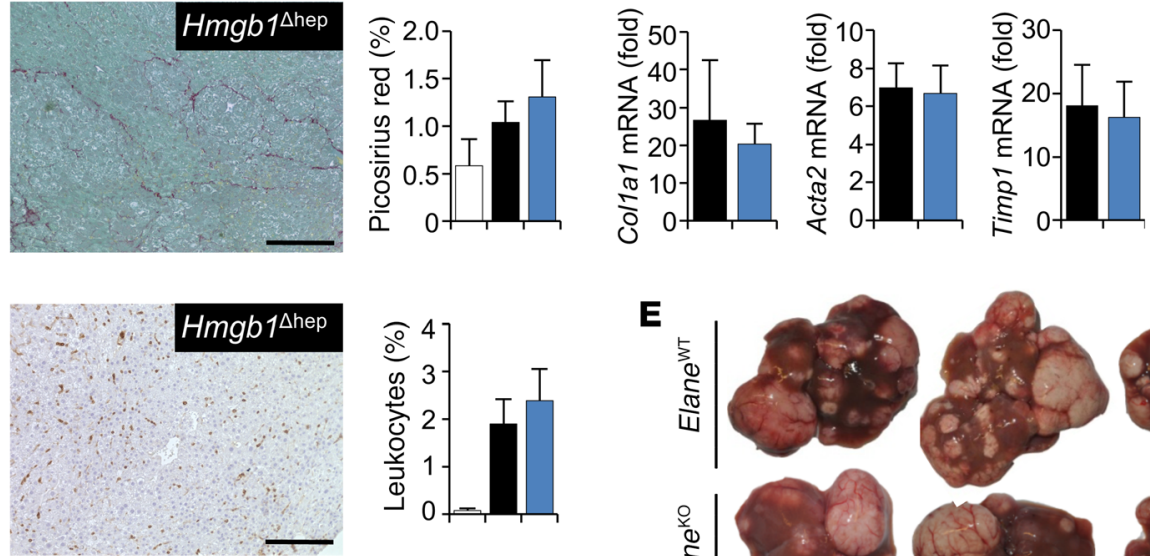

E
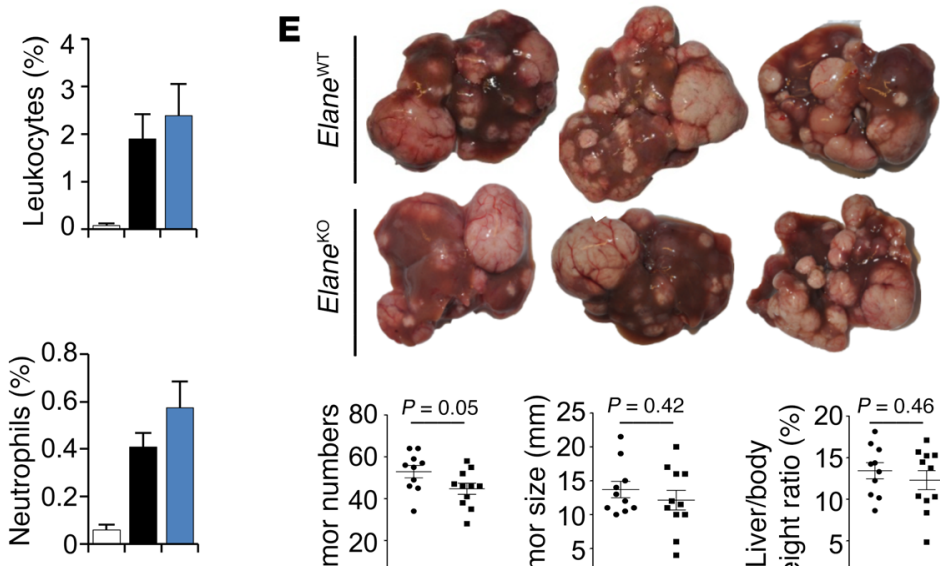
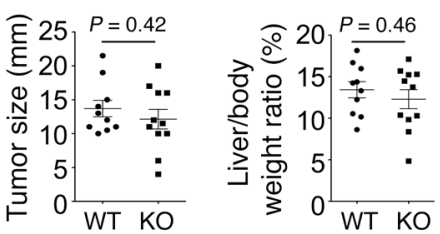

Figure 8. HMGB1 does not regulate fibrosis, proliferation, or inflammation in HCC. (A-D) Tumors from Hmgb $7^{\text {fl/fl }}$ mice $(n=12)$ and $H m g b 1^{\text {Ahep }}$ mice $(n=$ 12) treated with DEN plus 15 injections of $\mathrm{CCl}_{4}$ were analyzed by IHC and qPCR to determine proliferation (A), fibrosis (B), infiltrating CD45 leukocytes, (C) and Ly6B' neutrophils (D). (E) Hepatocarcinogenesis was induced in male WT $(n=10)$ and neutrophil elastase-deficient $\left(E l a n e^{\mathrm{KO}}, n=11\right)$ mice using the combination of DEN and 15 injections of $\mathrm{CCl}_{4}$, followed by quantification of tumor numbers, size, and liver/body weight ratios. Data are expressed as the mean \pm SEM. qPCR data are shown as the fold induction compared with normal liver. Statistical significance was determined by unpaired, 2 -tailed $t$ test. Scale bars: $100 \mu \mathrm{m}$.

have defective neutrophil effector functions but display normal neutrophil recruitment $(41,63)$. However, we found no significant contribution of neutrophil activation to HCC development, with similar tumor numbers, sizes, and liver/body weight ratios in WT and Elane-deficient littermates (Figure 8E), suggesting that neutrophil effector functions are not required for DEN plus $\mathrm{CCl}_{4}$ induced hepatocarcinogenesis. Moreover, we did not observe differences in the recruitment of $\mathrm{CD}^{+} \mathrm{T}$ cells (Supplemental Figure 11D), a cell population that is able to mediate antitumor immune responses or genotoxic stress, as determined by H2AX staining (Supplemental Figure 11E). Additionally, we did not observe a reduction in YAP protein or Yap1 or Wwtr1 mRNA levels and even detected an increase in TAZ protein levels in $H m g b 1^{\text {shep }}$ mice (Supplemental Figure 12A). Accordingly, YAP target genes, as determined by qPCR (Supplemental Figure 12B) or RNA sequencing (RNA-seq) (Supplemental Table 1), were either similar in $\mathrm{Hmgbl}^{\mathrm{H} / \mathrm{I}}$ and $H m g b 1^{\text {shep }}$ mice or higher in $H m g b 1^{\text {shep }}$ mice. In summary, our data demonstrate that the promotion of HCC by HMGB1 is not explained by effects on inflammation, antitumor responses, regeneration, fibrogenesis, or genotoxic stress, or by HMGB1- mediated modulation of YAP and TAZ. To better understand the influence of HMGB1 on hepatocarcinogenesis and follow up on the above-described finding that HMGB1 promotes the ductular metaplasia of hepatocytes (Figure 6), we performed RNA-seq in tumors from $H m g b 1^{\text {shep }}$ and $H m g b 1^{1 / / 1 / 1}$ mice treated with DEN plus $\mathrm{CCl}_{4}$. Unsupervised clustering of differentially expressed genes showed that tumors from $H m g b 1^{\text {shep }}$ mice clustered closer with normal liver than tumors from $\mathrm{Hmgb}^{\mathrm{t} / \mathrm{l} / \mathrm{l}}$ mice (Figure 9A and Supplemental Table 1). Accordingly, 675 of 848 genes (79.6\%) in tumors from $\mathrm{Hmgb}^{\text {shep }}$ mice had expression levels in the direction of normal liver $\left(P<2.2 \times 10^{-16}\right)$. A more mature phenotype of tumors from $H m g b 1^{\text {thep }}$ mice was further supported by the strong reduction in the oncofetal and progenitor markers Cd133, H19, and $A f p$ in $H m g b 1^{\text {shep }}$ tumors in our RNA-seq data (Supplemental Table 1), which was confirmed by qPCR and immunohistochemical AFP and A6 staining (Figure 9, B-D). These findings are consistent with clinical findings, in which HMGB1 serum levels were increased in HCC patients and strongly correlated with AFP levels (64). As we had previously shown that A6-positive tumor cells within HCC are derived from hepatocytes (26), our present find- 


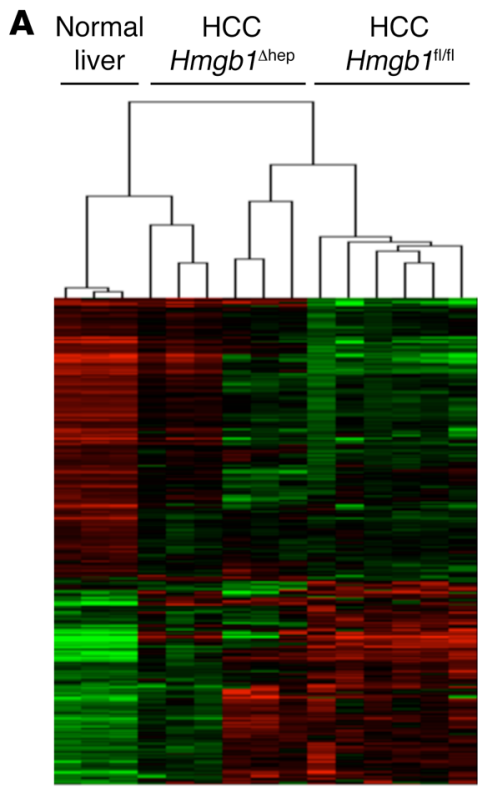

B
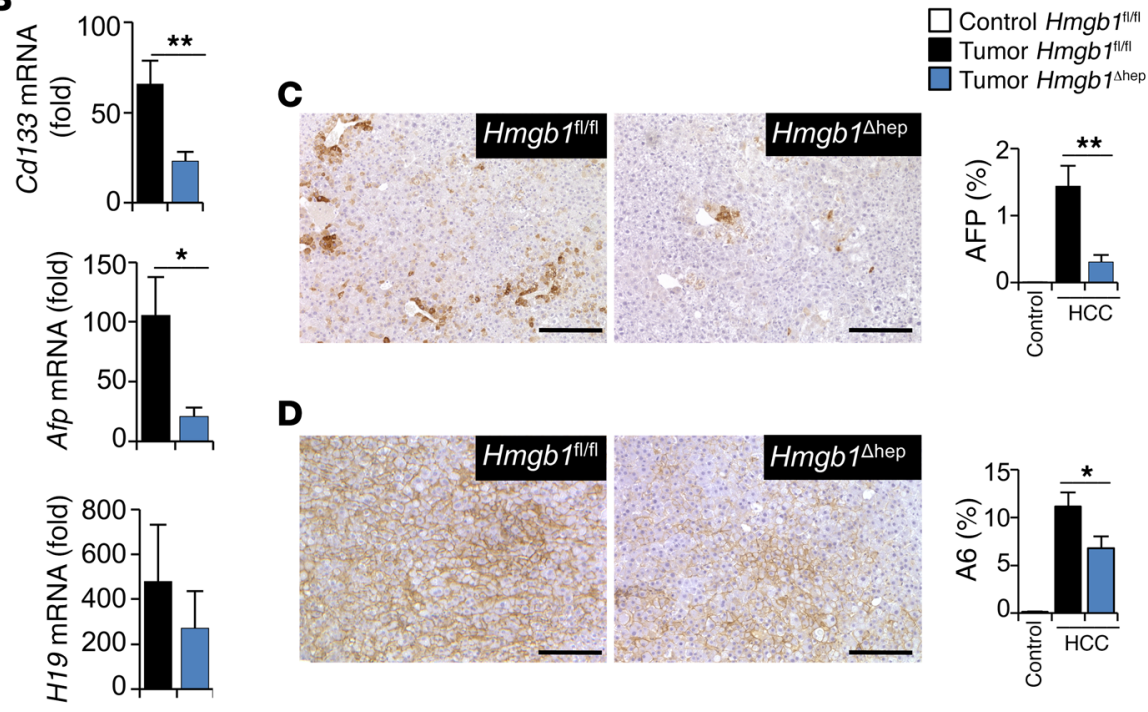

Figure 9. HMGB1 promotes tumor dedifferentiation and the progenitor signature. Tumors from $\mathrm{Hmg} g \mathrm{f}^{\mathrm{fl} / \mathrm{fl}}(n=6)$ and $\mathrm{Hmg} b \mathrm{1}^{\text {hhep }}(n=6)$ mice treated with DEN plus 15 injections of $\mathrm{CCl}_{4}$ as well as healthy control livers $(n=3)$ were subjected to RNA-seq. (A) Heatmap shows genes in the most relevant pathways, as described in Methods. (B) Expression of Cd133, Afp, and H19 was confirmed by qPCR. (C and D) AFP (C) and A6 (D) expression were determined by IHC in $\mathrm{Hmgb}^{\mathrm{fl} / \mathrm{fl}}(n=7-12)$ and $\mathrm{Hmgb}^{\text {thep }}(n=10-12)$ tumors. Data are expressed as the mean \pm SEM. qPCR data are shown as the fold induction compared with normal liver. ${ }^{*} P<0.05$ and ${ }^{*} P<0.01$, by unpaired, 2 -tailed $t$ test. Scale bars: $100 \mu \mathrm{m}$.

ings demonstrate that HMGB1 is a key pathway driving metaplasia and the progenitor signature in hepatocytes and hepatocytederived tumor cells. Notably, the presence of progenitor markers is one the most characteristic features of dedifferentiated HCC, and a large body of literature has demonstrated a positive correlation with worse clinical outcomes (29-36). As we had identified a key role for the HMGB1 receptor RAGE in driving ductular reactions in CLD, we next investigated the role of RAGE in HCC development and observed a significant reduction in tumor development in Rage ${ }^{\mathrm{KO}}$ mice in the DEN plus $\mathrm{CCl}_{4}$ model, as demonstrated by a reduction of tumor numbers, size, and liver/body weight ratios (Supplemental Figure 13).

\section{Discussion}

Cell death is a key component of CLD and considered an important driver of progression to fibrosis, cirrhosis, and HCC (3). Although DAMPs, including HMGB1, contribute to sterile inflammation in the setting of acute liver injury $(38,41,65)$, their role in other injury responses and contribution to CLD remain enigmatic. Our data implicate HMGB1 as an important hepatocyte DAMP that regulates specific cell death responses in the chronically injured liver. Our findings suggest that the main effect of HMGB1 in CLD is the promotion of ductular reactions, whereas other injury responses such as regeneration, inflammation, or fibrosis are not significantly affected. Given that we observed a strong but incomplete suppression of ductular reactions in $H m g b 1^{\text {shep }}$ mice, HMGB1 represents a key contributor for the efficient execution of this response but is not the only driver. This is consistent with findings that pathways such as $\beta$-catenin $(66,67), \mathrm{CCN} 1$ (68), and FGF signaling (69) also promote ductular reactions in the liver. Moreover, in our study, $\mathrm{CCl}_{4}$-induced liver injury did not trigger a sig- nificant progenitor response, despite increased HMGB1 release. Together, these findings indicate that HMGB1 release is necessary but not sufficient to trigger an effective progenitor response in the liver. Our data from two injury models with AAV8-TBG-Cremediated hepatocyte-specific HMGB1 deletion also exclude the possibility that the lack of intracellular HMGB1 in the ductular compartment may have impaired their ability to respond to injury. Of note, the majority of A6-positive ductular cells were not derived from hepatocytes, indicating that hepatocyte-derived HMGB1 acts in a nonautonomous fashion on other cell types to drive ductular reactions. As such, we found that HMGB1 was able to trigger Erk activation and proliferation in BMOL progenitor cells in vitro, suggesting that the effects of HMGB1 on ductular reactions in the injured liver are direct. In conjunction with our finding that HMGB1-deleted mice had a lower amount of Ki67-positive progenitor ductular cells and an extensive phospho-pathway screen in HMGB1-treated BMOL progenitor cells, these data suggest that HMGB1 stimulates ductular expansion predominantly via Erk activation and proliferation. Our in vivo data in $T l r 4^{\mathrm{KO}}, T l r 9^{\mathrm{KO}}$, and Rage ${ }^{\mathrm{KO}}$ mice and in vitro studies with RAGE, TLR4, and TLR9 inhibitors demonstrate that HMGB1 contributes to the ductular reaction via RAGE. Using liposomal clodronate, we excluded macrophages as a target that may indirectly mediate progenitor responses, but we cannot completely rule out the possibility that HMGB1 may additionally affect progenitors through indirect mechanisms that involve other cell types. Our studies using ethyl pyruvate also showed that active secretion of HMGB1 is not a key driver in DDC-induced ductular reactions, further emphasizing the key role of HMGB1 released from dying cells in this setting. These data are further supported by our finding that secreted, i.e., the acetylated form of disulfide HMGB1 was less abundant than 
the nonacetylated form in DDC-mediated injury. However, it is conceivable that secretion of acetylated HMGB1 is more abundant and may drive ductular reactions in other settings.

Although hepatocytes are the primary source for liver regeneration in multiple mouse models including those involving DDC or $\mathrm{CDE}$ diets, $\mathrm{CCl}_{4}$ injection, and partial hepatectomy $(15,16)$, these models do not achieve efficient suppression of hepatocyte proliferation and may not be ideal to test the contribution of ductular cells (14). Recent studies in novel mouse models $(21,22)$ as well as data from zebrafish $(19,20)$ demonstrated a key contribution of the ductular compartment to liver regeneration in settings in which hepatocyte proliferation is efficiently blocked. Accordingly, Lgr5-positive liver stem cells can generate hepatocytes in vitro and in vivo (23). However, it remains unclear whether HMGB1-mediated ductular cell expansion contributes to hepatocyte generation from this source and whether this may represent a regenerative mechanism in the setting of severe injury. Further studies of HMGB1 in models with efficient suppression of hepatocyte proliferation in combination with positive lineage tracing of ductular cells will be required to answer this question.

The second key finding of our study was the reduction of HCC in mice with liver-specific HMGB1 deletion. Hepatocellular death is a risk factor for $\operatorname{HCC}$ development $(3,6,7)$, and our data suggest that HMGB1 may provide a molecular link between cell death and HCC development in the chronically injured liver. This hypothesis is supported by our finding that HMGB1 did not significantly affect HCC development in a mouse model that lacks chronic cell death and subsequent HMGB1 release, whereas HCC development was blocked in 3 different HCC models with chronic cell death. These data not only demonstrate the key role of HMGB1 as a tumor-promoting DAMP in the setting of chronic injury, but also exclude the possibility that reduced HCC development was caused by the loss of intracellular HMGB1. As with our data on HMGB1 in ductular reactions, we found that RAGE was the most likely candidate receptor through which HMGB1 mediated its effect on hepatocarcinogenesis. This finding is consistent with those of a published study using the $M d r 2^{\mathrm{KO}}$ model, in which Rage ${ }^{\mathrm{KO}}$ mice developed less HCC (56). Together, our findings suggest that the HMGB1/RAGE signaling axis provides a molecular link between cell death and hepatocarcinogenesis and is likely a key component of maladaptive wound-healing responses, which are geared toward repairing the injured liver but become maladaptive and increase the risk for HCC development in the long term. Importantly, the gene expression tumors from $\mathrm{Hmgb}^{\text {thep }}$ mice were more similar to normal liver, and expressed lower levels of progenitor and oncofetal markers than tumors from $\mathrm{Hmgbl}^{\mathrm{f} / \mathrm{fl}}$ mice. High expression of these markers, reflecting poorly differentiated and stem cell-enriched tumors, has been correlated with poor clinical prognosis in a wide body of literature (29-36). Notably, serum HMGB1 levels are strongly increased in HCC patients and correlate with AFP levels as well as tumor size (64). Progenitor cells do not give rise to HCC in a large number of murine models, including the DEN plus $\mathrm{CCl}_{4}$ and DEN plus DDC models used in the current study $(26,27)$. As $\mathrm{CCl}_{4}$-induced liver injury is not associated with the development of ductular reactions, it is likely that HMGB1 induces protumorigenic signals (e.g., in the DEN plus $\mathrm{CCl}_{4}$ model) directly in preneoplastic or tumor cells. Given the findings of a study showing that tumor-forming cells, which are hepatocyte derived in DEN-induced HCC, express progenitor markers such as A6, CK19, H19, Epcam, and Sox9 (28), it is conceivable that HMGB1-mediated ductular metaplasia could increase the tumor-initiating capacity of hepatocytes. Future studies using metaplastic hepatocyte tracing are needed to determine whether these cells are more tumorigenic and how deletion of the HMGB1/RAGE signaling axis affects tumor formation from this cellular source. In addition, HMGB1-mediated increases of progenitor markers within already established tumors could promote more aggressive tumor behavior, as demonstrated by the strong clinical correlation between the progenitor signature and clinical outcomes (29-36). Further studies are required to determine these different possibilities and to identify downstream signals through which HMGB1 contributes to hepatocarcinogenesis and tumor dedifferentiation. In contrast to a recent study that reported HMGB1-regulated transcriptional activation of YAP in HCC (70), we did not observe a role for HMGB1 in regulating YAP or TAZ mRNA or protein expression or the expression of YAP target genes in HCC. This is consistent with our previous studies in normal liver (44), in which microarray analysis did not reveal differences in the expression of Yap1, Wwtr1 (encoding TAZ), or the YAP target genes Ankrd1, Ctgf, Cyr61, or Spp1, and is consistent with the fact that YAP and TAZ activity are predominantly regulated through posttranslational modification and protein stability rather than through transcription. Regardless of the underlying mechanism by which HMGB1 promotes hepatocarcinogenesis, our data suggest that interfering with this pathway could delay HCC development in patients with CLD. Glycyrrhizin, a phytochemical that inhibits HMGB1 (71), is widely used for the treatment of liver disease in Asia, and there are indications that glycyrrhizin can reduce HCC development in certain patient subsets (72).

Our study also demonstrates that hepatocellular HMGB1 is not a "master" DAMP that regulates all wound-healing responses in the chronically injured liver. As such, we did not observe an important contribution of HMGB1 in cell death responses such as fibrogenesis, inflammation, and proliferation. However, we observed a reduction of neutrophil recruitment in some models and a trend toward reduced neutrophil recruitment in other models in early stages of chronic liver injury. These findings are consistent with our previous findings on HMGB1/RAGE-mediated neutrophil recruitment in acute injury (41) but suggest that other pathways are more potent regulators of neutrophil recruitment in CLD. In contrast to a recent study on the role of HMGB1 in liver regeneration following acetaminophen intoxication (73), we did not observe a major role for HMGB1 in liver regeneration, except for a reduction of proliferation in the DDC diet model. In addition to using a different model, Tirone et al. (73) administered recombinant nonoxidizable HMGB1, whereas we deleted HMGB1. Thus, it is likely that the effects of HMGB1 on liver regeneration are model and redox dependent. Our results on the role of HMGB1 in liver fibrosis also differ from those of a recently published study (74). Our findings were based on 3 different models (Tak1 $1^{\text {shep }}, M d r 2^{\mathrm{KO}}$, and the DDC diet) and were additionally confirmed in the $\mathrm{MCDE}$ diet model and in $\mathrm{CCl}_{4}$-induced liver injury (data not shown). Moreover, liver-specific deletion of HMGB1 in mice with hepatic autophagy deficiency due to loss of ATG7 also 
did not show alterations in liver fibrosis (75), further confirming our data. Together, these findings suggest that additional cell death pathways, possibly mediated by other DAMPs or apoptotic bodies, must link cell death and fibrosis in the chronically injured liver. In summary, our findings suggest that DAMPs mediate cell death responses in a context-specific and a cell type-specific manner and that multiple branches of the hepatic wound-healing response are most likely regulated through a variety of DAMPs rather than a single master DAMP.

\section{Methods}

Mice. Mice were maintained on a 12-hour dark/12-hour light cycle with ad libitum access to food and water. $H m g b 1^{\mathrm{t} / \mathrm{l} l}$ mice $(41,44)$ were backcrossed with C57Bl/ 6 background at least 5 times. C57BL/6 mice, albumin-Cre mice, the Cre reporter line TdTomato (stock no. 007914), Tlr $4^{\mathrm{KO}}$ mice (stock no. 007227), and Tak1 $1^{\mathrm{f} / \mathrm{l} l}$ mice (stock no. 011039) were purchased from The Jackson Laboratory. Rage $\mathrm{KO}^{\mathrm{KO}}$ mice were a gift of Ann-Marie Schmidt (New York University, New York, New York, USA). $M d r 2^{\mathrm{KO}}$ mice (on a FVB background) were obtained from Detlef Schuppan (University of Mainz, Mainz, Germany). For conditional knockout of Hmgb1 in the Tak1 $1^{\text {hhep }}$ model, Tak1 ${ }^{1 / / 1}$ or Tak $1^{\text {t1/fl }}$ and $H m g b 1^{\mathrm{t} / \mathrm{ll}}$ double-floxed mice expressing albumin-Cre were bred with floxed or double-floxed mice negative for albumin-Cre. For conditional deletion of $H m g b 1$ in $M d r 2^{\mathrm{KO}}$ mice, $H m g b 1^{\mathrm{t} / \mathrm{l} \mathrm{l}}$ mice expressing albumin-Cre were backcrossed 3 times with $M d r 2^{\mathrm{KO}}$, followed by further interbreeding of the $\mathrm{F} 3$ generation.

HCC induction and evaluation. HCC was induced by a combination of $\mathrm{DEN}$ and $\mathrm{CCl}_{4}$ injections for the majority of experiments. Male offspring were injected with a single i.p. dose of DEN $(25 \mathrm{mg} / \mathrm{kg}$ body weight, MilliporeSigma) on postpartum day 15. Four weeks later, mice were treated with 15 weekly i.p injections of $\mathrm{CCl}_{4}(0.5 \mathrm{ml} / \mathrm{g}$ body weight, dissolved in corn oil at a ratio of 1:3, MilliporeSigma) and sacrificed 8-10 weeks after the last $\mathrm{CCl}_{4}$ injection. Some mice were fed a DDC diet for 4 months starting 4 weeks after receiving DEN and sacrificed 8 months after the initial DEN injection. As nongenotoxic

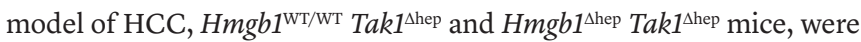
sacrificed at 9 months of age. As a pure genotoxic model of HCC, some mice were treated with a single dose of DEN $(25 \mathrm{mg} / \mathrm{kg}$, on postpartum day 15) and sacrificed 11 months later. For quantification, HCC tumor numbers and the largest tumor sizes were determined by counting the number of visible tumors (exceeding $1 \mathrm{~mm}$ in diameter) and measuring the size of the largest tumor with a caliper, respectively (57).

Chronic liver injury models. Chronic liver injury was induced in 8- to 10 -week-old male mice by feeding them a $0.1 \%$ DDC diet or an MCD diet supplemented with $0.15 \%$ ethionine for 3 weeks and 2 weeks, respectively. For selective deletion of HMGB1 in hepatocytes, $H m g b 1^{\mathrm{t} / \mathrm{fl}}$ mice were injected i.v. with $10^{11}$ genome copies of AAV8-TBG-Cre or AAV8-TBG-LacZ (58) on day 15 postpartum (for the $M d r 2^{\mathrm{KO}}$ mice) or 6 weeks postpartum (for all other mice). For some experiments, mice fed a DDC diet were treated with ethyl pyruvate $(40 \mathrm{mg} / \mathrm{kg}$, i.p., given twice weekly throughout the entire time mice were fed DDC diet). For some experiments, mice fed a DDC diet were treated with liposomal clodronate or control liposomes $(4 \mu \mathrm{l} / \mathrm{g}$ body weight, i.p., both from Liposoma), on days 4, 8, and 12 after starting the diet.

Partial hepatectomy and acute $\mathrm{CCl}_{4}$-induced liver injury. A twothirds partial hepatectomy was performed as described previously (76). Acute liver injury and subsequent hepatocyte proliferation were induced by a single i.p. injection of $\mathrm{CCl}_{4}(0.5 \mathrm{ml} / \mathrm{kg}$ body weight, dissolved in corn oil at a ratio of 1:3).

Hepatocyte tracing. To trace hepatocytes and determine whether HMGB1 deletion alters the presence of hepatocyte-derived ductular cells, mice that were either WT or $H m g b 1^{\mathrm{fl} / \mathrm{fl}}$ and also expressed the Cre reporter TdTomato were injected with $10^{11}$ genome copies of AAV8-TBG-Cre. One week later, mice were fed a DDC diet for 3 weeks. Subsequently, ductular metaplasia of hepatocytes was determined in liver sections by identification of TdTom-positive cells coexpressing A6, Sox9, or OPN.

IHC and immunofluorescence. Paraffin-embedded or frozen liver sections were stained with primary antibodies directed against A6 (77) (a gift of Valentina Factor, NIH); AFP (Proteintech, catalog 14550-1-AP); pan-cytokeratin (Dako, catalog Z0622); cytokeratin 19 (Developmental Studies Hybridoma Bank [DSHB] at the University of Iowa, Troma III); Ki67 (Abcam, catalog ab16667); Ly-6B.2 (AbD Serotec, clone MCA771G); F4/80 (AbD Serotec, clone CI:A3-1, cata$\log$ MCA497R); CD3 (Thermo Fisher Scientific, catalog RM-9107-S0); CD45 (BD Pharmingen, catalog 550539); YAP (Abcam, catalog ab205270); p-histone H3 (Ser10) (Santa Cruz Biotechnology, catalog sc-8656); Sox9 (MilliporeSigma, catalog AB5535); and OPN (R\&D Systems, catalog AF808). Detection was performed using either a fluorescent secondary antibody with various fluorescent conjugates (all from Thermo Fisher Scientific; chicken anti-goat, A21467; donkey anti-rabbit, A21206 or A21207; donkey anti-rat, A21208) or the Vectastain ABC HRP Kit (Vector Laboratories) with DAB or VIP substrate, followed by counterstaining with either DAPI (Thermo Fisher Scientific, D1306) or hematoxylin or methyl green. Fluorescence images were taken on a Nikon A1 confocal laser microscope. Nonfluorescence images were taken on an Olympus microscope coupled to a Retiga camera (Q Imaging). Morphometric quantification of immunohistochemical staining was done using Adobe Photoshop, with the exception of quantification of hepatocyte and progenitor cell proliferation, which was done using Fiji software. For quantification of hepatocyte proliferation, size and circularity thresholds were set so that nuclei of nonhepatocyte cells were not counted. For some experiments, IHC was performed with both fluorescence and a VIP substrate, with fluorescence images displayed as representative images and quantification done using VIP staining.

Western blot analysis and phospho-kinase screening. Western blotting for the detection of p-Erk was done using a primary mouse antibody against YAP/TAZ (Cell Signaling Technology, catalog 8418). Membranes were reprobed with HRP-conjugated GAPDH (MilliporeSigma, catalog G9295). After detection of p-Erk (Thr202/Tyr204) (Cell Signaling Technology, catalog 9106), membranes were stripped and relabeled with a rabbit antibody against Erk (Cell Signaling Technology, catalog 4695). Phospho-kinase screening was performed using a phospho-kinase array (R\&D Systems, ARY003B) according to the manufacturer's instructions.

RNA isolation and qPCR. RNA was isolated from cells and tissues by column purification and on-column DNAse treatment (Roche Diagnostics). Following reverse transcription, qPCR was performed using primer-probe pairs (Applied Biosystems) and relative standard curves as described previously (57).

Analysis of HMGB1 by electrospray ionization liquid chromatography tandem mass spectrometry. Posttranslational modifications of HMGB1 were detected in mouse plasma with liquid chromatography tandem 
mass spectrometry (LC-MS/MS) using an AB Sciex QTRAP 5500 or an AB Sciex TripleTOF 5600 equipped with a NanoSpray II source by in-line LC and an U3000 HPLC System connected to a $180 \mu \mathrm{m} \times$ $20 \mathrm{~mm}$ nanoACQUITY UPLC C18 trap column and a $75 \mu \mathrm{m} \times 15 \mathrm{~cm}$ nanoACQUITY UPLC BEH130 C18 column (Waters) using previously described conditions $(41,78)$.

RNA-seq and bioinformatics. RNA (RNA integrity number [RIN] $>8$, as determined by Bioanalyzer 2100, Agilent Technologies) from normal liver HCC $(n=3)$ or HCC from $\operatorname{Hmgb1}^{\mathrm{fl} / \mathrm{fl}}(n=6)$ or $H m g b 1^{\text {shep }}$ $(n=6)$ mice was used to construct libraries with the Illumina TruSeq RNA Preparation Kit according to the manufacturer's instructions. Thirty million 100-bp single-end sequencing was performed on the Illumina 2500 at the JP Sulzberger Columbia Genome Center (New York, New York, USA). These data were deposited in the Gene Expression Omnibus database (GEO GSE89689). Differential expression was determined between both $H m g b 1^{\text {shep }} \mathrm{HCC}$ and $H m g b 1^{1 / / 1 /} \mathrm{HCC}$ and between $H m g b 1^{1 / / 1 l}$ HCC and normal liver. Counts were normalized with the trimmed mean of M values method (TMM) (79). Differential expression was estimated using weighted Limma-Voom, with a significance cutoff of the Benjamini-Hochberg FDR of 0.05 or less, an absolute $\log _{2}$ fold change of 0.6 or greater, and restriction to the 12 most significant pathways as determined by iPathwayGuide, plus the progenitor and oncofetal genes Afp, H19, and Prom1. Both identification and calculation were performed in R. TMM-normalized counts of these genes were transformed by $\log _{2}$ (counts +0.5 ) centered, clustered with average linkage clustering using cluster 3.0, and displayed with Java TreeView (http://jtreeview.sourceforge.net/). The statistical significance of genes from Hmgb1 $1^{\text {shep }}$ HCC that showed expression in the direction of normal liver versus those that did not was calculated with the binomial test.

Cell lines and cell culture experiments. BMOL cells (provided by George Yeoh, University of Western Australia, Perth, Australia) (51) were seeded in 12-well or 24-well plates in William's E medium (Thermo Fisher Scientific) supplemented with 10\% FBS (Gemini), recombinant mouse EGF (10 ng/ml, Thermo Fisher Scientific), and human recombinant insulin $(10 \mu \mathrm{g} / \mathrm{ml}$, Thermo Fisher Scientific). Cells were treated next day with either disulfide or a fully reduced form of HMGB1 (80) for the indicated durations (Figure 5, B-F and H; Supplemental Figure 8D; and Supplemental Figure 9, D and E). Cells were then lysed for gene expression and Western blot analysis. When indicated, the cells were pretreated for 30 minutes with the MEK inhibitor UO126 (MilliporeSigma). To determine proliferation, cells were grown in media con- taining $1 \%$ FBS and growth factors, followed by addition of HMGB1 and an MTT assay (Invitrogen, Thermo Fisher Scientific). For some experiments, cells were pretreated with anti-RAGE $(5 \mu \mathrm{g} / \mathrm{ml}$, Santa Cruz Biotechnology, catalog sc-365154) or an isotype control ( $5 \mu \mathrm{g} / \mathrm{ml}$, Santa Cruz Biotechnology, sc-3877); anti-TLR4 (5 $\mu \mathrm{g} / \mathrm{ml}$, InvivoGen, catalog mab-mtlr4md2) or an isotype control $(5 \mu \mathrm{g} / \mathrm{ml}$, InvivoGen, catalog mabg2a-ctlrt); and TLR9 antagonist (ODN 2088, $1 \mu \mathrm{M}$, InvivoGen, catalog tlrl-2088) or a control nucleotide (ODN 2088 control, InvivoGen, catalog tlrl-2088c) for 8 hours, followed by treatment with HMGB1.

Study approval. All animal procedures were performed with the approval of the IACUC of Columbia University and were in accordance with the Guide for the Care and Use of Laboratory Animals (National Academies Press, 2011).

Statistics. All data are expressed as the mean \pm SEM. An unpaired, 2 -tailed $t$ test was used for comparisons of 2 groups.

\section{Author contributions}

$\mathrm{CH}$ designed and performed in vivo experiments on liver injury and hepatocarcinogenesis and in vitro experiments, analyzed data, and wrote the manuscript. PH designed and performed in vivo experiments related to liver injury and hepatocarcinogenesis and analyzed data. JPP performed in vivo experiments related to hepatocarcinogenesis. RAF analyzed RNA-seq data and performed bioinformatics analysis. DJA coordinated HMGB1 bioanalysis and recombinant HMGB1 production. RFS conceptualized and oversaw all aspects of the study, designed experiments, analyzed data, and wrote the manuscript.

\section{Acknowledgments}

We would like to thank Geum Youn Gwak (Sungkyunkwan University School of Medicine, Seoul, Korea), who contributed to the generation of $H m g b 1^{\mathrm{t} / \mathrm{ll}}$ mice; George Yeoh (University of Western Australia); Bin Gao (NIH, Bethesda, Maryland, USA) for providing BMOL cells; and Valentina Factor (NIH) for providing the A6 antibody. This study was supported by funding from the NIH (R01CA200597, 1R01DK116620, and U01AA021912, to RFS), 5P30CA013696 (Columbia University Cancer Center support grant), and a fellowship from the German Research Foundation (Hu 1953/1-1).

Address correspondence to: Robert F. Schwabe, 1130 St. Nicholas Avenue, ICRC, Room 926, New York, New York 10032, USA. Phone: 212.851.5462; Email: rfs2102@cumc.columbia.edu.
1. Lozano R, et al. Global and regional mortality from 235 causes of death for 20 age groups in 1990 and 2010: a systematic analysis for the Global Burden of Disease Study 2010. Lancet. 2012;380(9859):2095-2128.

2. El-Serag HB. Hepatocellular carcinoma. N Engl J Med. 2011;365(12):1118-1127.

3. Luedde T, Kaplowitz N, Schwabe RF. Cell death and cell death responses in liver disease: mechanisms and clinical relevance. Gastroenterology. 2014;147(4):765-783.e4.

4. Tai DI, Lin SM, Sheen IS, Chu CM, Lin DY, Liaw YF. Long-term outcome of hepatitis B e antigen-negative hepatitis B surface antigen carriers in relation to changes of alanine aminotransferase levels over time. Hepatology. 2009;49(6):1859-1867.
5. Ghany MG, et al. Progression of fibrosis in chronic hepatitis C. Gastroenterology. 2003;124(1):97-104.

6. Chen CF, et al. Changes in serum levels of HBV DNA and alanine aminotransferase determine risk for hepatocellular carcinoma. Gastroenterology. 2011;141(4):1240-1248.e1.

7. Lee $\mathrm{MH}$, et al. Hepatitis $\mathrm{C}$ virus seromarkers and subsequent risk of hepatocellular carcinoma: longterm predictors from a community-based cohort study. JClin Oncol. 2010;28(30):4587-4593.

8. Boege $\mathrm{Y}$, et al. A dual role of caspase-8 in triggering and sensing proliferation-associated DNA damage, a key determinant of liver cancer development. Cancer Cell. 2017;32(3):342-359.e10.

9. Ruhl CE, Everhart JE. Elevated serum alanine aminotransferase and gamma-glutamyltrans- ferase and mortality in the United States population. Gastroenterology. 2009;136(2):477-85.e11.

10. Bettermann K, et al. TAK1 suppresses a NEMOdependent but NF-kappaB-independent pathway to liver cancer. Cancer Cell. 2010;17(5):481-496.

11. Hikita H, et al. Bak deficiency inhibits liver carcinogenesis: a causal link between apoptosis and carcinogenesis. J Hepatol. 2012;57(1):92-100.

12. Luedde T, et al. Deletion of NEMO/IKKgamma in liver parenchymal cells causes steatohepatitis and hepatocellular carcinoma. Cancer Cell. 2007;11(2):119-132.

13. Weber A, et al. Hepatocyte-specific deletion of the antiapoptotic protein myeloid cell leukemia-1 triggers proliferation and hepatocarcinogenesis in mice. Hepatology. 2010;51(4):1226-1236. 
14. Michalopoulos GK, Khan Z. Liver stem cells: experimental findings and implications for human liver disease. Gastroenterology. 2015;149(4):876-882.

15. Malato Y, et al. Fate tracing of mature hepatocytes in mouse liver homeostasis and regeneration. JClin Invest. 2011;121(12):4850-4860.

16. Yanger K, et al. Adult hepatocytes are generated by self-duplication rather than stem cell differentiation. Cell Stem Cell. 2014;15(3):340-349.

17. Schaub JR, Malato Y, Gormond C, Willenbring H. Evidence against a stem cell origin of new hepatocytes in a common mouse model of chronic liver injury. Cell Rep. 2014;8(4):933-939.

18. Jörs $\mathrm{S}$, et al. Lineage fate of ductular reactions in liver injury and carcinogenesis. JClin Invest. 2015;125(6):2445-2457.

19. Choi TY, Ninov N, Stainier DY, Shin D. Extensive conversion of hepatic biliary epithelial cells to hepatocytes after near total loss of hepatocytes in zebrafish. Gastroenterology. 2014;146(3):776-788.

20. He J, Lu H, Zou Q, Luo L. Regeneration of liver after extreme hepatocyte loss occurs mainly via biliary transdifferentiation in zebrafish. Gastroenterology. 2014;146(3):789-800.e8.

21. Lu WY, et al. Hepatic progenitor cells of biliary origin with liver repopulation capacity. Nat Cell Biol. 2015;17(8):971-983.

22. Raven A, et al. Cholangiocytes act as facultative liver stem cells during impaired hepatocyte regeneration. Nature. 2017;547(7663):350-354.

23. Huch $\mathrm{M}$, et al. Long-term culture of genomestable bipotent stem cells from adult human liver. Cell. 2015;160(1-2):299-312.

24. Yanger K, et al. Robust cellular reprogramming occurs spontaneously during liver regeneration. Genes Dev. 2013;27(7):719-724.

25. Tarlow BD, et al. Bipotential adult liver progenitors are derived from chronically injured mature hepatocytes. Cell Stem Cell. 2014;15(5):605-618.

26. Mu X, et al. Hepatocellular carcinoma originates from hepatocytes and not from the progenitor/biliary compartment. J Clin Invest. 2015;125(10):3891-3903.

27. Shin S, et al. Genetic lineage tracing analysis of the cell of origin of hepatotoxin-induced liver tumors in mice. Hepatology. 2016;64(4):1163-1177.

28. He G, et al. Identification of liver cancer progenitors whose malignant progression depends on autocrine IL-6 signaling. Cell. 2013;155(2):384-396.

29. Lee JS, et al. A novel prognostic subtype of human hepatocellular carcinoma derived from hepatic progenitor cells. Nat Med.2006;12(4):410-416.

30. Yamashita T, et al. EpCAM and alphafetoprotein expression defines novel prognostic subtypes of hepatocellular carcinoma. Cancer Res. 2008;68(5):1451-1461.

31. Yang XR, et al. High expression levels of putative hepatic stem/progenitor cell biomarkers related to tumour angiogenesis and poor prognosis of hepatocellular carcinoma. Gut. 2010;59(7):953-962.

32. Villanueva A, et al. Combining clinical, pathology, and gene expression data to predict recurrence of hepatocellular carcinoma. Gastroenterology. 2011;140(5):1501-1512.e2.

33. Kim H, et al. Human hepatocellular carcinomas with "Stemness"-related marker expression: keratin 19 expression and a poor prognosis. Hepa- tology. 2011;54(5):1707-1717.

34. Ji J, Wang XW. Clinical implications of cancer stem cell biology in hepatocellular carcinoma. Semin Oncol. 2012;39(4):461-472.

35. Won C, et al. Signal transducer and activator of transcription 3-mediated CD133 up-regulation contributes to promotion of hepatocellular carcinoma. Hepatology. 2015;62(4):1160-1173.

36. Agopian VG, et al. Evaluation of patients with hepatocellular carcinomas that do not produce $\alpha$-fetoprotein. JAMA Surg. 2017;152(1):55-64.

37. Lotze MT, et al. The grateful dead: damageassociated molecular pattern molecules and reduction/oxidation regulate immunity. Immunol Rev. 2007;220:60-81.

38. Kubes P, Mehal WZ. Sterile inflammation in the liver. Gastroenterology. 2012;143(5):1158-1172.

39. Hernandez C, Huebener P, Schwabe RF. Damage-associated molecular patterns in cancer: a double-edged sword. Oncogene. 2016;35(46):5931-5941.

40. Vénéreau E, Ceriotti C, Bianchi ME. DAMPs from cell death to new life. Front Immunol. 2015;6:422.

41. Huebener $\mathrm{P}$, et al. The HMGB1/RAGE axis triggers neutrophil-mediated injury amplification following necrosis. JClin Invest. 2015;125(2):539-550.

42. Mariotti V, Strazzabosco M, Fabris L, Calvisi DF. Animal models of biliary injury and altered bile acid metabolism. Biochim Biophys Acta. 2018;1864(4 Pt B):1254-1261.

43. Trauner M, Fickert P, Wagner M. MDR3 (ABCB4) defects: a paradigm for the genetics of adult cholestatic syndromes. Semin Liver Dis. 2007;27(1):77-98

44. Huebener P, et al. High-mobility group box 1 is dispensable for autophagy, mitochondrial quality control, and organ function in vivo. Cell Metab. 2014;19(3):539-547.

45. Bonaldi T, et al. Monocytic cells hyperacetylate chromatin protein $\mathrm{HMGB} 1$ to redirect it towards secretion. EMBO J. 2003;22(20):5551-5560.

46. Scaffidi P, Misteli T, Bianchi ME. Release of chromatin protein HMGB1 by necrotic cells triggers inflammation. Nature. 2002;418(6894):191-195.

47. Davé SH, et al. Ethyl pyruvate decreases HMGB1 release and ameliorates murine colitis. JLeukoc Biol. 2009;86(3):633-643.

48. Kim YM, Park EJ, Kim JH, Park SW, Kim HJ, Chang KC. Ethyl pyruvate inhibits the acetylation and release of HMGB1 via effects on SIRT1/ STAT signaling in LPS-activated RAW264.7 cells and peritoneal macrophages. Int Immunopharmacol. 2016;41:98-105.

49. Boulter L, et al. Macrophage-derived Wnt opposes Notch signaling to specify hepatic progenitor cell fate in chronic liver disease. Nat Med. 2012;18(4):572-579.

50 . Venereau E, et al. Mutually exclusive redox forms of HMGB1 promote cell recruitment or proinflammatory cytokine release. J Exp Med. 2012;209(9):1519-1528.

51. Tirnitz-Parker JE, Tonkin JN, Knight B, Olynyk JK, Yeoh GC. Isolation, culture and immortalisation of hepatic oval cells from adult mice fed a choline-deficient, ethionine-supplemented diet. Int J Biochem Cell Biol. 2007;39(12):2226-2239.

52. Yimlamai $D$, et al. Hippo pathway activity influences liver cell fate. Cell. 2014;157(6):1324-1338.
53. Zhou D, et al. Mst1 and Mst2 maintain hepatocyte quiescence and suppress hepatocellular carcinoma development through inactivation of the Yap1 oncogene. Cancer Cell. 2009;16(5):425-438.

54. Kemper K, et al. Mutations in the Ras-Raf Axis underlie the prognostic value of CD133 in colorectal cancer. Clin Cancer Res. 2012;18(11):3132-3141.

55. Tabu K, et al. Analysis of an alternative human CD133 promoter reveals the implication of Ras/ ERK pathway in tumor stem-like hallmarks. Mol Cancer. 2010;9:39.

56. Pusterla T, et al. Receptor for advanced glycation endproducts (RAGE) is a key regulator of oval cell activation and inflammationassociated liver carcinogenesis in mice. Hepatology. 2013;58(1):363-373.

57. Dapito DH, et al. Promotion of hepatocellular carcinoma by the intestinal microbiota and TLR4. Cancer Cell. 2012;21(4):504-516.

58. $\mathrm{Mu} \mathrm{X}$, et al. Epithelial transforming growth factor- $\beta$ signaling does not contribute to liver fibrosis but protects mice from cholangiocarcinoma. Gastroenterology. 2016;150(3):720-733.

59. Inokuchi S, et al. Disruption of TAK1 in hepatocytes causes hepatic injury, inflammation, fibrosis, and carcinogenesis. Proc Natl Acad Sci U S A. 2010;107(2):844-849.

60. Wilson CL, et al. NFkB1 is a suppressor of neutrophil-driven hepatocellular carcinoma. Nat Commun. 2015;6:6818.

61. Templeton AJ, et al. Prognostic role of neutrophilto-lymphocyte ratio in solid tumors: a systematic review and meta-analysis. J Natl Cancer Inst. 2014;106(6):dju124.

62. Li YW, et al. Intratumoral neutrophils: a poor prognostic factor for hepatocellular carcinoma following resection. J Hepatol. 2011;54(3):497-505.

63. Belaaouaj A, et al. Mice lacking neutrophil elastase reveal impaired host defense against gram negative bacterial sepsis. Nat Med. 1998;4(5):615-618.

64. Cheng BQ, et al. Serum high mobility group box chromosomal protein 1 is associated with clinicopathologic features in patients with hepatocellular carcinoma. Dig Liver Dis. 2008;40(6):446-452.

65. McDonald B, et al. Intravascular danger signals guide neutrophils to sites of sterile inflammation. Science. 2010;330(6002):362-366.

66. Apte U, Thompson MD, Cui S, Liu B, Cieply B, Monga SP. Wnt/beta-catenin signaling mediates oval cell response in rodents. Hepatology. 2008;47(1):288-295.

67. Hu M, et al. Wnt/beta-catenin signaling in murine hepatic transit amplifying progenitor cells. Gastroenterology. 2007;133(5):1579-1591.

68. Kim KH, Chen CC, Alpini G, Lau LF. CCN1 induces hepatic ductular reaction through integrin $\alpha v \beta_{5}$-mediated activation of NF-kB. JClin Invest. 2015;125(5):1886-1900.

69. Utley S, et al. Fibroblast growth factor signaling regulates the expansion of A6-expressing hepatocytes in association with AKT-dependent $\beta$-catenin activation. JHepatol. 2014;60(5):1002-1009.

70. Chen R, et al. High mobilitygroup protein B1 controls liver cancer initiation through yesassociated protein-dependent aerobic glycolysis. Hepatology. 2018;67(5):1823-1841.

71. Mollica L, et al. Glycyrrhizin binds to high- 
mobility group box 1 protein and inhibits its cytokine activities. Chem Biol. 2007;14(4):431-441.

72. Veldt BJ, Hansen BE, Ikeda K, Verhey E, Suzuki $\mathrm{H}$, Schalm SW. Long-term clinical outcome and effect of glycyrrhizin in 1093 chronic hepatitis C patients with non-response or relapse to interferon. Scand J Gastroenterol. 2006;41(9):1087-1094.

73. Tirone M, et al. High mobility group box 1 orchestrates tissue regeneration via CXCR4. J Exp Med. 2018;215(1):303-318.

74. Arriazu E, et al. Signalling via the osteopontin and high mobility group box-1 axis drives the fibrogenic response to liver injury. Gut. 2017;66(6):1123-1137.
75. Khambu B, et al. HMGB1 promotes ductular reaction and tumorigenesis in autophagydeficient livers [published online ahead of print March 20, 2018]. J Clin Invest. https://doi. org/10.1172/JCI91814.

76. Mitchell C, Willenbring H. A reproducible and well-tolerated method for $2 / 3$ partial hepatectomy in mice. Nat Protoc. 2008;3(7):1167-1170.

77. Engelhardt NV, Factor VM, Medvinsky AL, Baranov VN, Lazareva MN, Poltoranina VS. Common antigen of oval and biliary epithelial cells (A6) is a differentiation marker of epithelial and erythroid cell lineages in early development of the mouse. Differentiation. 1993;55(1):19-26. 78. Antoine DJ, et al. Molecular forms of HMGB1 and keratin-18 as mechanistic biomarkers for mode of cell death and prognosis during clinical acetaminophen hepatotoxicity. J Hepatol. 2012;56(5):1070-1079.

79. Robinson MD, Oshlack A. A scaling normalization method for differential expression analysis of RNA-seq data. Genome Biol. 2010;11(3):R25.

80. Yang H, et al. Redox modification of cysteine residues regulates the cytokine activity of high mobility group box-1 (HMGB1). Mol Med. 2012;18:250-259. 